\title{
A comparison of staggered solution schemes for coupled particle-continuum systems modeled with the Arlequin method
}

\author{
S. Pfaller • G. Possart • P. Steinmann • M. Rahimi • F. Müller-Plathe • \\ M. C. Böhm
}

Received: date / Accepted: date

\begin{abstract}
This contribution aims at a systematic investigation of staggered solution schemes for the computation of coupled domains having different resolutions in space, a problem frequently arising in multiscale modeling of materials. To couple a standard finite element domain with a high resolution atomistic or coarse-grained, i.e. particle-based domain, a so-called bridging domain is considered. In this handshake region a total energy, which is the sum of the weighted energies of both domains, needs to be formulated. Interactions in the particle domain are modeled by potential functions, e.g. a harmonic potential in the simplest case or the LENNARD-JONES potential to consider also anharmonic interactions between the particles. The main goal is to separate the computation of finite element and particle domains as much as possible, amongst others to calculate the different domains on several CPUs.

In the present work, the governing equations of the coupling method are presented. The energy functions of continuum, particle domain and bridging domain are recapitulated and the coupling constraint is set up. For
\end{abstract}

S. Pfaller, G. Possart, P. Steinmann

Chair of Applied Mechanics, University of Erlangen-Nuremberg,

Egerlandstrasse 5, 91058 Erlangen, Germany

Tel.: +49 (0)9131-85-28502

Fax: +49 (0)9131-85-28503

E-mail: pfaller@ltm.uni-erlangen.de

E-mail: possart@ltm.uni-erlangen.de

E-mail: steinmann@ltm.uni-erlangen.de

M. Rahimi, F. Müller-Plathe, M. C. Böhm

Theoretical Physical Chemistry, Darmstadt University of Technology, Petersenstr. 20, 64285 Darmstadt, Germany

Tel.: +49 (0)6151 166523

Fax: +49 (0)6151 166526

E-mail: f.mueller-plathe@theo.chemie.tu-darmstadt.de

E-mail: boehm@theo.chemie.tu-darmstadt.de

E-mail: m.rahimi@theo.chemie.tu-darmstadt.de the sake of simplicity, these relations are reformulated for the case of a one dimensional system. On the one hand, this system is computed monolithically without any separation of domains. On the other hand, various staggered solution schemes are derived systematically. The relevant equations of each scheme are given in detail together with the sequent iteration steps. All staggered schemes are investigated qualitatively, e.g. by their convergence behavior, as well as quantitatively by comparing the staggered solutions with the monolithic solution.

Keywords atomistic-continuum coupling · multiscale modeling · bridging domain method · domain decomposition · Lagrange multipliers

\section{Introduction and outline}

Continuum mechanics usually treats matter as homogeneous and neglects its molecular or atomistic structure, respectively. This approach is very well suited for modeling bodies and systems whose macroscopic size is much larger than the dimensions on the microscopic level, e.g. interatomic distances.

Many material properties, however, are caused by the atomistic structure of matter. This has to be taken into account for numerous applications, like e.g. cracks, crazes, etc. In these cases, it is inevitable to model materials at the level of particles in terms of their interaction potentials, the simplest options being represented by only pairwise interactions of harmonic or LennardJones type. To simulate such particle systems, Molecular Dynamics (MD) or Monte Carlo (MC) solution techniques are usually applied. Although it is not difficult to set up the equations of motion for the particles, the large numbers of them required for technically relevant 
system sizes implies problems beyond the capability of present computers.

The above mentioned drawbacks can be attenuated by coupling particle based methods to continuum approaches, i.e. only crucial sections like crack tips or boundary layers, which require a high resolution, are modeled at particle level while the surrounding domains are treated as a continuum (e.g. linear or nonlinear elastic, viscoelastic, etc.) and are discretized and solved by the finite element method (FEM). In this contribution, a bridging domain method, the so called Arlequin method, is applied to realize the coupling. Other couplings like an edge-to-edge coupling as discussed e.g. in [4] shall not be considered here. Furthermore, electronic effects are not taken into account explicitly, although applications in this direction have been reported e.g. in [14].

The Arlequin method was introduced by Ben Dhia, cf. [5], [6] and [7]. Originally designed to couple FE domains of different resolutions, this method has been extended e.g. in [2] to couple atomistic and continuum models. Very similar to this approach is the method employed by Guidault and Belytschko in [12], which was also used in several modeling studies of carbon nanotubes, cf. e.g. [20] and [13].

Unlike the Quasicontinuum method proposed by Tadmor et al. [17], the Arlequin method does not just increase the resolution of the FE domain to that of the atomic lattice. Instead, a pure particle region can be defined without any underlying FE description. Furthermore, the Arlequin method does not require a particle lattice. Thus, it is also possible to model amorphous materials like polymers. Important work in this field has been done by Prudhomme et al. in publications on the modeling of generic polymer systems, cf. [3] and [9].

Beyond spatial coupling as described here, temporal coupling of methods is a major issue in the field of multiscale modeling. Thereby the problem consists in the big gap between time scales in the respective domains. FE simulations usually deal with time steps at the range of seconds, minutes, or even hours. In contrast, dynamic particle simulations are always bound to time scales that are directly related to the movements of particles. Typically, time steps at the range of nanoseconds or even femtoseconds have to be considered. This leads to a difference of ten to fifteen orders of magnitude and has to be handled very carefully. Important contributions in this field are, among others, [1], [18] and [19].

Hence, there is a big difference between particle methods and continuum based approaches with respect to length and time scales. Numerical simulations at the atomistic or coarse grained level usually deal with huge numbers of particles ranging from some ten thousands up to millions. To capture these numbers, particle methods require powerful computers as well as special schemes to treat large systems. When the coupling to FE with larger length and time scales is required, it is reasonable to keep the computations of the respective domains as separated as possible, i.e. to compute both problems iteratively by using staggered solution schemes. Thereby, the information transfer necessary for the staggered computation should be kept as simple and small as possible.

The aim of this contribution is to investigate a coupled one dimensional system and to give an overview of staggered solution schemes and their applicability. Important characteristics like e.g. convergence, computational performance, and degree of decoupling will be discussed.

\section{Modeling foundations}

In this section, a short overview of the basics of continuum mechanical and particle based modeling is given. Starting from the mathematical formulations in the respective domains, the governing equations for their coupling by the Arlequin method will furthermore be summarized.

\subsection{Continuum modeling}

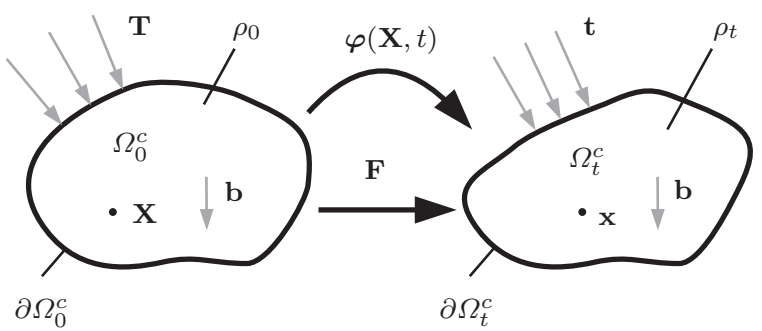

Fig. 1 Continuum mechanical setting: deformation map $\varphi$ from material configuration $\Omega_{0}^{c}$ to spatial configuration $\Omega_{t}^{c}$; quantities are defined in the text

In continuum mechanics, a vector-valued function

$\varphi: \mathbf{X} \mapsto \mathbf{X}=\varphi(\mathbf{X}, t)$

is considered which maps the material configuration $\Omega_{0}^{c}$ of a continuous body to the spatial configuration $\Omega_{t}^{c}$ as sketched in Figure 1. X denotes a point in the undeformed material configuration, whereas $\mathbf{x}$ is the same point in the deformed spatial configuration. Furthermore, the deformation gradient

$\mathbf{F}=\frac{\partial \varphi}{\partial \mathbf{X}}=\frac{\partial}{\partial \mathbf{X}}(\mathbf{X}+\mathbf{u})=\mathbf{I}+\frac{\partial \mathbf{u}}{\partial \mathbf{X}}$ 
is introduced with displacement vector $\mathbf{u}=\mathbf{x}-\mathbf{X}$ and identity $\mathbf{I}$. In order to describe the deformation of a body, the internal potential energy

$E_{c}^{i n t}=\int_{\Omega_{0}^{c}} \Psi(\mathbf{F}) d V$

is used, whereby the scalar function $\Psi=\Psi(\mathbf{F})$ denotes the strain or stored energy density while $V$ is the volume in the material configuration. Every deformation of the body results in a change of the internal potential energy. In case of conservative systems as investigated here, there is no dissipation of energy due to the deformation.

Since it does not affect the main interests of this work, we will furthermore concentrate on linear elastic systems and small strains. Consequently, the stored energy density can be expressed in terms of the symmetric strain tensor

$\boldsymbol{\varepsilon}=\frac{1}{2}\left[\frac{\partial \mathbf{u}}{\partial \mathbf{X}}+\left(\frac{\partial \mathbf{u}}{\partial \mathbf{X}}\right)^{T}\right]$

and the material constants $\lambda$ and $\mu$ via

$\Psi(\varepsilon)=\frac{\lambda}{2}[\varepsilon: \mathbf{I}]^{2}+\mu\left[\varepsilon^{2}: \mathbf{I}\right]$.

The external energy introduced to the body is expressed in terms of surface tractions $\mathbf{T}$ and body forces $\mathbf{b}$. With $d A$ denoting area elements in the material description we obtain

$E_{c}^{e x t}=\int_{\partial_{\sigma} \Omega_{0}^{c}} \mathbf{u} \cdot \mathbf{T} d A+\int_{\Omega_{0}^{c}} \mathbf{u} \cdot \rho_{0} \mathbf{b} d V$.

In (6), the Neumann boundary $\partial_{\sigma} \Omega_{0}^{c}$ is assumed to be separated from the DiRICHLET boundary $\partial_{\mathbf{u}} \Omega_{0}^{c}$, i.e.

$\partial_{\sigma} \Omega_{0}^{c} \cap \partial_{\mathbf{u}} \Omega_{0}^{c}=\varnothing$.

The total potential energy of a static system thus follows as

$E_{c}^{t o t}=E_{c}^{i n t}-E_{c}^{e x t}$.

The mechanical system is equilibrated if $E_{c}^{t o t}$ takes its global minimum.

\subsection{Particle statics}

In contrast to continuum mechanics, a particle system as sketched in Figure 2 is a discrete system containing a number $n_{d}$ of point masses. The interactions between particles are described by potential functions $E_{d}^{i n t}$, which can be specified e.g. as pair potentials, torsion potentials, and angle-bending potentials. Again for

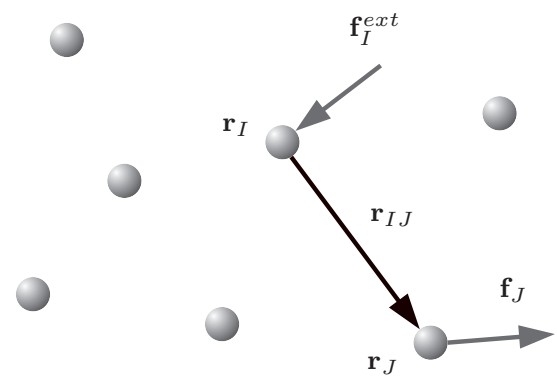

Fig. 2 Particle system: current distance vector $\mathbf{r}_{I J}$ between particles $I$ and $J$, sum of internal forces $\mathbf{f}_{J}$ acting on particle $J$, external force $\mathbf{f}_{I}^{e x t}$ acting on particle $I$

the sake of simplicity, this paper only considers pairwise interactions:

$E_{I J}=\tilde{E}_{I J}\left(\mathbf{r}_{I}, \mathbf{r}_{J}\right)$

Here, index $I$ refers to particle $I$ at a current position $\mathbf{r}_{I}$, whereas index $J$ refers to particle $J$ at $\mathbf{r}_{J}$. Similar to the formulation in continuum mechanics, a displacement vector $\mathbf{w}_{I}$ can be introduced as the difference between initial position $\mathbf{R}_{I}$ and current position $\mathbf{r}_{I}$ of particle $I$ :

$\mathbf{w}_{I}=\mathbf{r}_{I}-\mathbf{R}_{I}$

The interaction potential can then be expressed in terms of positions as well as in terms of displacements:

$\tilde{E}_{I J}\left(\mathbf{r}_{I}, \mathbf{r}_{J}\right)=E_{I J}\left(\mathbf{w}_{I}, \mathbf{w}_{J}\right)$.

The internal energy of the whole particle system coincides with the sum over the energy contributions $E_{I J}$ of all bonds or the sum of the energy contributions $E_{I}$ of all particles, respectively, i.e.

$$
E_{d}^{i n t}=\frac{1}{2} \sum_{I=1}^{n_{d}} \sum_{\substack{J=1 \\ J \neq I}}^{n_{d}} E_{I J}\left(\mathbf{w}_{I}, \mathbf{w}_{J}\right)=\sum_{I=1}^{n_{d}} E_{I}\left(\mathbf{w}_{I}, \mathbf{w}_{J}\right)
$$

with

$$
E_{I}\left(\mathbf{w}_{I}, \mathbf{w}_{J}\right)=\frac{1}{2} \sum_{\substack{J=1 \\ J \neq I}}^{n_{d}} E_{I J}\left(\mathbf{w}_{I}, \mathbf{w}_{J}\right)
$$

The force $\mathbf{f}_{I J}$ acting on particle $I$ due to the interaction with particle $J$ is the negative gradient of the interaction potential with respect to the position vector $\mathbf{r}_{I}$ :

$\mathbf{f}_{I J}=-\frac{\partial E_{I J}}{\partial \mathbf{r}_{I}}$. 
The total force on a certain particle due to its interaction with all other particles follows as

$\mathbf{f}_{I}=\sum_{\substack{J=1 \\ J \neq I}}^{n_{d}} \mathbf{f}_{I J}$

Up to now, the interaction potentials have not been specified. Depending on the material under consideration, harmonic and anharmonic interaction potentials can be chosen. Harmonic potentials are used e.g. to describe the interaction between adjacent atoms of a molecule while anharmonic potentials model further interactions, e.g. between atoms of different molecules. The simplest realization is the harmonic potential, which reads

$E_{I J}^{H}=\frac{1}{2} k\left|\mathbf{w}_{I}-\mathbf{w}_{J}\right|^{2}$

and is governed by the force constant $k$. The more sophisticated and frequently applied LENNARD-JONES potential

$E_{I J}^{L J}=4 \epsilon\left[\left(\frac{\sigma}{r_{I J}}\right)^{12}-\left(\frac{\sigma}{r_{I J}}\right)^{6}\right]$

is formulated in terms of the potential depth $\epsilon$, the characteristic length $\sigma$ and the absolute value $r_{I J}=\left|\mathbf{r}_{I J}\right|=$ $\left|\mathbf{r}_{I}-\mathbf{r}_{J}\right|$. This potential is often used to consider nonbonded interactions as well as anharmonic effects.

The external energy of a particle system follows from the external forces applied and the corresponding displacements:

$E_{d}^{e x t}=\sum_{I=1}^{n_{d}} \mathbf{f}_{I}^{e x t} \cdot \mathbf{w}_{I}$.

According to (8), the total potential energy of the static particle systems follows:

$E_{d}^{t o t}=E_{d}^{i n t}-E_{d}^{e x t}$

Again, an equilibrium of this system is obtained at a minimum of $E_{d}^{t o t}$.

It is obvious that both approaches, the continuous as well as the discrete one, lead to very similar formulations of the respective energies. In particular, in both cases equilibrium is found by minimizing the total energies. This encourages employing a coupling method that is based on energy formulations.
2.3 Coupling continuum and particle statics: the Arlequin method

The method used here to couple continuum and particle domain was originally introduced as Arlequin method by Ben Dhia, cf. [5], [6] and [7]. This particular type of method is based on a blending of particle and continuum energies in a bridging domain by using a weighting factor. Furthermore, a coupling constraint is introduced to define a relation between the continuous displacement field and the discrete particle displacements.

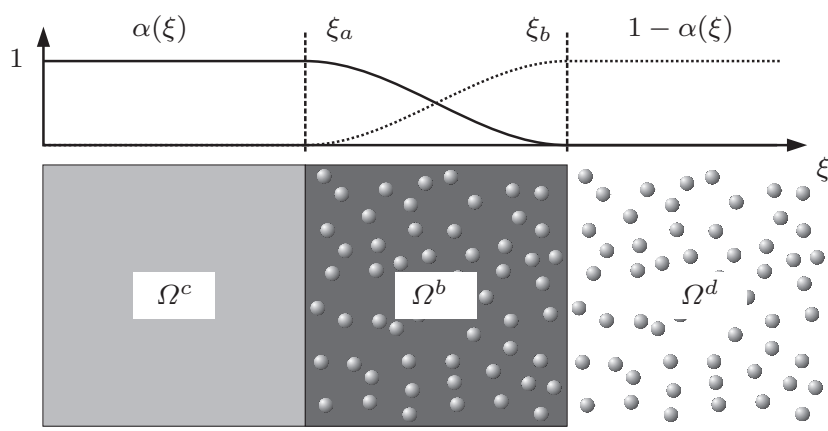

Fig. 3 Coupled system: continuum $\Omega^{c}$, bridging domain $\Omega^{b}$, particle domain $\Omega^{d}$, and weighting factor $\alpha(\xi)$

Figure 3 depicts this coupling scheme: a continuum $\Omega^{c}$ is coupled to a particle domain $\Omega^{d}$ by a bridging domain $\Omega^{b}$. The energies in the respective domains are scaled using a weighting factor $\alpha(\xi)$ with $\xi=\xi(\mathbf{X})$ in the continuum and $\xi=\xi\left(\mathbf{R}_{I}, \mathbf{R}_{J}\right)$ in the particle domain. The coupling factor satisfies the following conditions:

$\alpha(\xi)\left\{\begin{array}{ll}=1 & \text { in } \Omega^{c} \backslash \Omega^{b} \\ \in[0,1] & \text { in } \Omega^{b} \\ =0 & \text { in } \Omega^{d} \backslash \Omega^{b}\end{array}\right.$.

According to [2] and [10], it can be chosen to be constant, linear, cubic or a trigonometric function in $\Omega_{b}$ :

$\alpha(\xi)=\frac{1}{2}$,

$\alpha(\xi)=1-\frac{\xi-\xi_{a}}{\xi_{b}-\xi_{a}}$,

$\alpha(\xi)=\frac{-\left[\xi-\xi_{b}\right]^{2}\left[2 \xi-3 \xi_{a}+\xi_{b}\right]}{\left[\xi_{a}-\xi_{b}\right]^{3}}$,

$\alpha(\xi)=1-\cos ^{2}\left(\frac{\xi_{b}-\xi}{2\left[\xi_{b}-\xi_{a}\right]} \pi\right)$,

where $\xi_{a}$ and $\xi_{b}$ denote the values of $\xi$ at the interface between continuum and bridging domain as well as between particle domain and bridging domain, respectively. 


\subsection{Energy functions}

Using factor $\alpha$, the weighted energies in the respective domains read as follows:

continuum:

$\hat{E}_{c}^{i n t}=\int_{\Omega_{0}^{c}} \alpha(\xi(\mathbf{X})) \Psi(\mathbf{F}) d V$

$\hat{E}_{c}^{e x t}=\int_{\partial_{\sigma} \Omega_{0}^{c}} \alpha(\xi(\mathbf{X})) \mathbf{u} \cdot \mathbf{T} d A+\int_{\Omega_{0}^{c}} \alpha(\xi(\mathbf{X})) \mathbf{u} \cdot \rho_{0} \mathbf{b} d V$

particle domain:

$\hat{E}_{d}^{i n t}=\frac{1}{2} \sum_{I=1}^{n_{d}} \sum_{\substack{J=1 \\ J \neq I}}^{n_{d}}\left[1-\alpha\left(\xi\left(\mathbf{R}_{I}, \mathbf{R}_{J}\right)\right)\right] E_{I J}\left(\mathbf{w}_{I}, \mathbf{w}_{J}\right)$

$\hat{E}_{d}^{e x t}=\sum_{I=1}^{n_{d}}\left[1-\alpha\left(\xi\left(\mathbf{R}_{I}\right)\right)\right] \mathbf{f}_{I}^{e x t} \cdot \mathbf{w}_{I}$

$\alpha\left(\xi\left(\mathbf{R}_{I}, \mathbf{R}_{J}\right)\right)$ and $\alpha\left(\xi\left(\mathbf{R}_{I}\right)\right)$ will later be specified in detail. The total energy of the coupled static system is then given as the sum of the weighted energies of the continuum and of the particle domain, i.e.

$\hat{E}^{t o t}=\hat{E}_{c}^{i n t}+\hat{E}_{d}^{i n t}-\hat{E}_{c}^{e x t}-\hat{E}_{d}^{e x t}$.

\subsection{Coupling constraint}

In addition to the blending of energies, a coupling constraint is required to ensure matching of continuum and particle displacements in the bridging domain. According to [2], this match of displacements is realized by evaluating the difference between the continuous displacement field $\mathbf{u}$ and an artificial displacement field $\mathbf{w}^{*}$ in the particle domain following from an interpolant $\Pi$, which will be specified later, via

$\mathbf{w}^{*}=\Pi \mathbf{w}$.

This kind of workaround is necessary since there is no displacement field available in the particle domain but only discrete displacements. In (27), particle displacements and positions are summarized as follows:

$\mathbf{R}=\left\{\mathbf{R}_{1}, \cdots \mathbf{R}_{I}, \cdots \mathbf{R}_{n_{d}}\right\}^{T}$
$\mathbf{r}=\left\{\mathbf{r}_{1}, \cdots \mathbf{r}_{I}, \cdots \mathbf{r}_{n_{d}}\right\}^{T}$
$\mathbf{w}=\mathbf{r}-\mathbf{R}$.
Using $\mathbf{w}^{*}$, the following coupling constraint is defined to enforce matching displacements:

$$
\begin{aligned}
\| \mathbf{u} & -\mathbf{w}^{*} \|^{2}=\beta_{1} \int_{\Omega_{b}}\left[\mathbf{u}-\mathbf{w}^{*}\right] \cdot\left[\mathbf{u}-\mathbf{w}^{*}\right] d V \\
& +\beta_{2} \int_{\Omega_{b}} \frac{\partial}{\partial \mathbf{X}}\left[\mathbf{u}-\mathbf{w}^{*}\right]: \frac{\partial}{\partial \mathbf{X}}\left[\mathbf{u}-\mathbf{w}^{*}\right] d V \stackrel{!}{=} 0 .
\end{aligned}
$$

Equation (29) refers to the $L^{2}$ norm in case of $\left(\beta_{1}, \beta_{2}\right)=$ $(1,0)$ and to the $H^{1}$ norm for $\left(\beta_{1}, \beta_{2}\right)=(1,1)$. To minimize total energy (26) of the coupled system and to enforce the coupling constraint, the Lagrange multiplier method is applied. Using Lagrange multipliers $\boldsymbol{\lambda}$, the coupling constraint can be rewritten to

$$
\begin{aligned}
b(\boldsymbol{\lambda}, \mathbf{u}, \mathbf{r}) & =\beta_{1} \int_{\Omega_{b}} \boldsymbol{\lambda} \cdot\left[\mathbf{u}-\mathbf{w}^{*}\right] d V \\
& +\beta_{2} \int_{\Omega_{b}} \frac{\partial}{\partial \mathbf{X}} \boldsymbol{\lambda}: \frac{\partial}{\partial \mathbf{X}}\left[\mathbf{u}-\mathbf{w}^{*}\right] d V
\end{aligned}
$$

Thus, the optimization problem can be solved by computing the saddle point of

$L(\boldsymbol{\lambda}, \mathbf{u}, \mathbf{r})=\hat{E}_{t o t}+b(\boldsymbol{\lambda}, \mathbf{u}, \mathbf{r})$.

\subsection{Reduction to one dimension}

Since this contribution is aiming at an evaluation of staggered schemes for the above coupled optimization problem, we will furthermore consider only the one dimensional case. For the sake of simplicity, the system of interest consists of a linear elastic rod representing the continuum $\Omega^{c}$, a chain of particles representing the particle domain $\Omega^{d}$, and an overlap region $\Omega^{b}$ as shown in Figure 4.

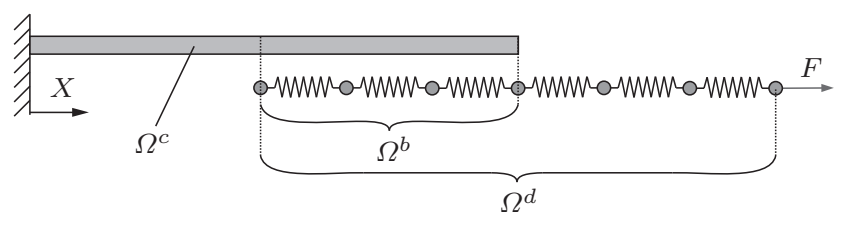

Fig. 4 Coupling of a continuum $\Omega^{c}$ (with Young's modulus $E$ and cross-sectional area $A$ ) and a particle domain $\Omega^{d}$. The bridging domain is denoted by $\Omega^{b}$.

The displacement field in the continuum is denoted by $u$, while the particle displacements $w_{I}$ are assembled into a vector $\mathbf{w}$. The rod is clamped at the left hand side, i.e. $u(0)=0$, and an external force $F$ is applied to the outer particle at the right hand side. In this simple case, the weighted internal energies of linear elastic continuum and particle domain reduce to

$\hat{E}_{c}^{i n t}=\int_{\Omega_{c}} \alpha(X) \frac{1}{2} E A u^{\prime}(X)^{2} d X$ 
and

$$
\hat{E}_{d}^{i n t}=\frac{1}{2} \sum_{I=1}^{n_{d}} \sum_{\substack{J=1 \\ J \neq I}}^{n_{d}}\left[1-\alpha\left(R_{I}, R_{J}\right)\right] E_{I J}\left(w_{I}, w_{J}\right) .
$$

The initial particle positions can be expressed in terms of the coordinate $X$ via $R_{I}=X_{I}$. Furthermore, it is not necessary to introduce the scalar variable $\xi$ for the weighting factor. Instead, the coordinate $X$ can be used directly in (21). According to [2], $\alpha\left(R_{I}, R_{J}\right)$ and $\alpha\left(R_{I}\right)$ can, therefore, be specified as follows:

$\alpha\left(R_{I}, R_{J}\right)=\alpha\left(\frac{X_{I}+X_{J}}{2}\right)$

$\alpha\left(R_{I}\right)=\alpha\left(X_{I}\right)$.

Note that definition (34) of $\alpha$ is not necessarily optimal as it may create additional errors when considering next-to-nearest neighbor interactions. More elaborate and consistent discrete weights have been discussed in the literature. The external energy of the continuum is zero, while that of the particle domain follows from the displacement $w_{n_{d}}$ of the outer particle:

$\hat{E}_{d}^{e x t}=F w_{n_{d}}$.

In this particular case, it is not necessary to weight the external energy since the external force is applied in the pure particle domain only.

\subsection{Discretization}

To solve optimization problem (31), it is mandatory to discretize the displacement field in the continuum as well as the Lagrange multiplier field, which is realized here using piecewise linear basis functions:

$u(X) \approx \sum_{i=1}^{n_{c}} N_{i}^{c}(X) u_{i}^{h}=\mathbf{N}^{c}(X) \cdot \mathbf{u}^{h}$,
$\lambda(X) \approx \sum_{j=1}^{n_{\lambda}} N_{j}^{\lambda}(X) \lambda_{j}^{h}=\mathbf{N}^{\lambda}(X) \cdot \lambda^{h}$.

The nodal values are denoted by $u_{i}^{h}$ and $\lambda_{j}^{h}$ with the respective basis functions $N_{i}$ and $N_{j}, n_{c}$ and $n_{\lambda}$ are the number of nodes in the discretized continuum and the Lagrange multiplier space, respectively. The nodal values are assembled into the vectors $\mathbf{u}^{h}$ and $\boldsymbol{\lambda}^{h}$.

Piecewise linear basis functions can also be used to interpolate the discrete particle displacements, i.e.

$w^{*}(X)=\Pi \mathbf{w}=\sum_{I=1}^{n_{d}} N_{I}^{d}(X) w_{I}=\mathbf{N}^{d}(X) \cdot \mathbf{w}$.
Thus, $L$ becomes a function of the discretized displacements and Lagrange multipliers:

$L(\boldsymbol{\lambda}, \mathbf{u}, \mathbf{r}) \approx L\left(\boldsymbol{\lambda}^{h}, \mathbf{u}^{h}, \mathbf{w}\right)$.

The necessary condition for a saddle point of (31) reads

$\delta_{\boldsymbol{\lambda}^{h}, \mathbf{u}^{h}, \mathbf{w}} L\left(\boldsymbol{\lambda}^{h}, \mathbf{u}^{h}, \mathbf{w}\right)=0$,

which leads to three sets of equations. The first is related to the FE domain and obtained from the derivative of $L$ with respect to the nodal displacements $\mathbf{u}^{h}$ :

$\nabla_{\mathbf{u}^{h}} L=0 \longrightarrow\left\{\begin{array}{ll}\hat{\mathbf{f}}_{c}^{i n t}\left(\mathbf{u}^{h}\right) & \hat{\mathbf{f}}_{c}^{e x t} \\ \mathbf{f}_{b}^{i n t}\left(\mathbf{u}^{h}\right)+\mathbf{f}^{c p l}\left(\boldsymbol{\lambda}^{h}\right) & =\mathbf{f}_{b}^{e x t}\end{array}\right\}$

$\hat{\mathbf{f}}_{c}^{\text {int }}$ and $\mathbf{f}_{b}^{\text {int }}$ are the internal force vectors in the pure FE domain and in the bridging domain, respectively, whereas $\mathbf{f}^{c p l}$ denotes the vector of coupling forces due to the coupling constraint. $\hat{\mathbf{f}}_{c}^{e x t}$ and $\mathbf{f}_{b}^{e x t}$ are the external force vectors in the respective domains. The internal force vectors can be written using a $\left(n_{c} \times n_{c}\right)$ stiffness matrix $\mathbf{K}^{c}$ in the continuum and a $\left(n_{c b} \times n_{\lambda}\right)$ coupling matrix $\mathbf{G}_{c \lambda}$ in the bridging domain, where $n_{c b}$ denotes the number of $\mathrm{FE}$ nodes in the bridging domain:

$$
\begin{aligned}
{\left[\begin{array}{l}
\hat{\mathbf{f}}_{c}^{i n t} \\
\mathbf{f}_{b}^{i n t}
\end{array}\right] } & =\mathbf{K}^{c} \cdot\left[\begin{array}{l}
\hat{\mathbf{u}}^{c} \\
\mathbf{u}^{b}
\end{array}\right]=\mathbf{K}^{c} \cdot \mathbf{u}^{h} \\
\mathbf{f}^{c p l} & =\mathbf{G}_{c \lambda} \cdot \boldsymbol{\lambda}^{h}
\end{aligned}
$$

Here, the vector of nodal displacements $\mathbf{u}^{h}$ is separated into a vector of nodal displacements $\hat{\mathbf{u}}^{c}$ in the pure $\mathrm{FE}$ domain and a vector of displacements $\mathbf{u}^{b}$ in the bridging domain.

Quite similarly, one obtains the system of equations related to the particle domain:

$$
\nabla_{\mathbf{w}} L=0 \longrightarrow\left\{\begin{array}{ll}
-\mathbf{g}_{b}^{i n t}(\mathbf{w})+\mathbf{g}^{c p l}\left(\boldsymbol{\lambda}^{h}\right) & =\mathbf{g}_{b}^{\text {ext }} \\
-\mathbf{g}_{d}^{\text {int }}(\mathbf{w}) & =\mathbf{g}_{d}^{\text {ext }}
\end{array}\right\}
$$

According to the notation above, $\mathbf{g}_{d}^{\text {int }}$ and $\mathbf{g}_{b}^{\text {int }}$ are the internal force vectors in the pure particle domain and in the bridging domain, respectively. $\mathbf{g}^{c p l}$ is the vector of coupling forces acting on the particles due to the coupling constraint, while $\mathbf{g}_{d}^{e x t}$ and $\mathbf{g}_{b}^{\text {ext }}$ denote the external force vectors in the respective domains. Since the investigations in this contribution are restricted to a harmonic potential, a $\left(n_{d} \times n_{d}\right)$ stiffness matrix $\mathbf{K}^{d}$ can be identified in the particle domain, whereas a $\left(n_{d b} \times n_{\lambda}\right)$ coupling matrix $\mathbf{G}_{d \lambda}$ is used to rewrite the coupling forces. In this case, $n_{d b}$ is the number of particles in the bridging domain:

$$
\begin{aligned}
{\left[\begin{array}{l}
\mathbf{g}_{b}^{\text {int }} \\
\mathbf{g}_{d}^{\text {int }}
\end{array}\right] } & =\mathbf{K}^{d} \cdot\left[\begin{array}{l}
\mathbf{w}^{b} \\
\mathbf{w}^{d}
\end{array}\right]=\mathbf{K}^{d} \cdot \mathbf{w} \\
\mathbf{g}^{c p l} & =\mathbf{G}_{d \lambda} \cdot \boldsymbol{\lambda}^{h}
\end{aligned}
$$


Again, the vector of displacements $\mathbf{w}$ is separated into a part $\mathbf{w}^{b}$ referring to the bridging domain and a second part $\mathbf{w}^{d}$ that is related to the pure particle domain.

Eventually, the gradient of $L$ with respect to the Lagrange multipliers leads to:

$\nabla_{\boldsymbol{\lambda}^{h}} L=0 \longrightarrow\left\{\mathbf{G}_{\lambda c} \cdot \mathbf{u}^{b}+\mathbf{G}_{\lambda d} \cdot \mathbf{w}^{b}=\mathbf{0}\right\}$

\section{Monolithic computation}

Considering DIRICHLET boundary conditions only for the pure FE domain, equations (42), (45) and (48) can be combined to

$$
\left[\begin{array}{llllll}
\mathbf{K}_{p p}^{c} & \mathbf{K}_{p c}^{c} & \mathbf{K}_{p b}^{c} & \mathbf{0} & \mathbf{0} & \mathbf{0} \\
\mathbf{K}_{c p}^{c} & \mathbf{K}_{c c}^{c} & \mathbf{K}_{c b}^{c} & \mathbf{0} & \mathbf{0} & \mathbf{0} \\
\mathbf{K}_{b p}^{c} & \mathbf{K}_{b c}^{c} & \mathbf{K}_{b b}^{c} & \mathbf{0} & \mathbf{0} & \mathbf{G}_{c \lambda} \\
\mathbf{0} & \mathbf{0} & \mathbf{0} & \mathbf{K}_{b b}^{d} & \mathbf{K}_{b d}^{d} & \mathbf{G}_{d \lambda} \\
\mathbf{0} & \mathbf{0} & \mathbf{0} & \mathbf{K}_{d b}^{d} & \mathbf{K}_{d d}^{d} & \mathbf{0} \\
\mathbf{0} & \mathbf{0} & \mathbf{G}_{\lambda c} & \mathbf{G}_{\lambda d} \mathbf{0} & \mathbf{0}
\end{array}\right] \cdot\left[\begin{array}{c}
\mathbf{u}_{p}^{c} \\
\mathbf{u}^{c} \\
\mathbf{u}^{b} \\
\mathbf{w}^{b} \\
\mathbf{w}^{d} \\
\boldsymbol{\lambda}^{h}
\end{array}\right]=\left[\begin{array}{l}
\mathbf{f}_{u}^{e x t} \\
\mathbf{f}_{c}^{e x t} \\
\mathbf{f}_{b}^{e x t} \\
\mathbf{g}_{b}^{e x t} \\
\mathbf{g}_{d}^{e x t} \\
\mathbf{0}
\end{array}\right]
$$

Here, force vectors and displacements in the pure FE domain are split into unknown and prescribed parts:

$\hat{\mathbf{f}}_{c}^{e x t}=\left[\begin{array}{l}\mathbf{f}_{u}^{e x t} \\ \mathbf{f}_{c}^{e x t}\end{array}\right], \hat{\mathbf{f}}_{c}^{i n t}=\left[\begin{array}{l}\mathbf{f}_{u}^{i n t} \\ \mathbf{f}_{c}^{\text {int }}\end{array}\right], \hat{\mathbf{u}}^{c}=\left[\begin{array}{l}\mathbf{u}_{p}^{c} \\ \mathbf{u}^{c}\end{array}\right]$.

The variables used in (49) and (50) denote the following quantities:

$$
\begin{array}{ll}
\mathbf{u}_{p}^{c} & \text { prescribed nodal displ. in pure FE domain } \\
\mathbf{u}^{c} & \text { unknown nodal displ. in pure FE domain } \\
\mathbf{u}^{b} & \text { nodal displacements in bridging domain } \\
\mathbf{w}^{b} & \text { particle displacements in bridging domain } \\
\mathbf{w}^{d} & \text { particle displacements in pure particle domain } \\
\boldsymbol{\lambda}^{h} & \text { Lagrange multipliers } \\
\mathbf{f}_{u}^{\text {ext }} & \text { reaction forces in pure FE domain } \\
\mathbf{f}_{c}^{\text {ext }} & \text { prescribed nodal forces in pure FE domain } \\
\mathbf{f}_{b}^{\text {ext }} & \text { nodal forces in bridging domain } \\
\mathbf{g}_{b}^{\text {ext }} & \text { particle forces in bridging domain } \\
\mathbf{g}_{d}^{\text {ext }} & \text { particle forces in pure particle domain }
\end{array}
$$

Furthermore, the stiffness matrices in the respective domains can be identified as follows:

$$
\mathbf{K}^{c}=\left[\begin{array}{ccc}
\mathbf{K}_{p p}^{c} & \mathbf{K}_{p c}^{c} & \mathbf{K}_{p b}^{c} \\
\mathbf{K}_{c p}^{c} & \mathbf{K}_{c c}^{c} & \mathbf{K}_{c b}^{c} \\
\mathbf{K}_{b p}^{c} & \mathbf{K}_{b c}^{c} & \mathbf{K}_{b b}^{c}
\end{array}\right], \mathbf{K}^{d}=\left[\begin{array}{ll}
\mathbf{K}_{b b}^{d} & \mathbf{K}_{b d}^{d} \\
\mathbf{K}_{d b}^{d} & \mathbf{K}_{d d}^{d}
\end{array}\right] .
$$

Superscript $c$ refers to the FE-domain, whereas $d$ is chosen for the stiffness matrix in the particle domain. In addition, the right hand subscript is linked to the respective displacement vector, whereas the left hand subscript is associated with the force vector. In particular, the product

$\mathbf{K}_{b p} \cdot \mathbf{u}_{p}^{c}$

contributes to the nodal forces $\mathbf{f}_{b}^{\text {ext }}$ in the bridging domain and

$\mathbf{K}_{c p} \cdot \mathbf{u}_{p}^{c}$

is part of the prescribed nodal forces $\mathbf{f}_{c}^{e x t}$ in the pure FE domain.

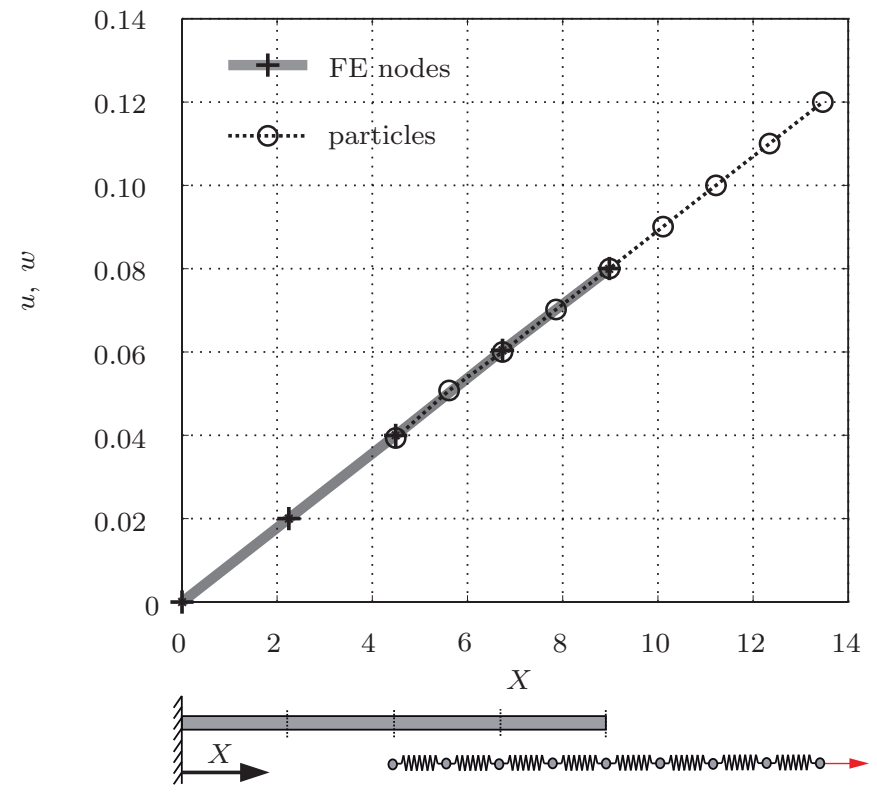

Fig. 5 Nodal displacements $\mathbf{u}$ and particle displacements $\mathbf{w}$ as a function of the coordinate $X$, variables chosen as follows: $\left[E A, k, F, l_{F E}, \beta_{1}, \beta_{2}, \alpha\right]=\left[6.41 \cdot 10^{-5} N, 5.71 \cdot 10^{-5} N / m m, 5.71\right.$. $10^{-7} \mathrm{~N}, 2.25 \mathrm{~mm}, 1,1$, cubic]

As an example, we consider a system containing two finite elements in the pure continuum, additionally two finite elements and five particles in the bridging domain as well as four particles in the pure particle domain. For the sake of simplicity, the Lagrange multipliers match with the FE nodes. The force constant $k$ and the stiffness $E A$ are chosen such that the stiffnesses of the rod and of the particle chain are the same. Furthermore, the $H^{1}$ norm $\left(\beta_{1}=1, \beta_{2}=1\right)$ and a cubic weighting function $\alpha$ are used. It has been reported in [2] that the resulting system of equations does not form a well-posed problem if the $L^{2}$ norm $\left(\beta_{1}=1, \beta_{2}=0\right)$ is chosen. In our experience, though, we did not encounter problems with the $L^{2}$ norm. Indeed, our examples do not change 
significantly if we employ the $L^{2}$ norm instead of the $H^{1}$ norm, also cf. the comparison in Section 5.

Eq. (49) can be solved in a monolithic way using a standard solver for systems of linear equations. The results for the displacements in the respective domains are plotted in Figure 5 and show a linear shape as expected.

\section{Staggered computation schemes}

While in the previous section the system of equations (49) was solved monolithically by using a standard solver, this section will focus on staggered solution schemes with separated computations in the respective domains. As it was already mentioned in the introduction, particle simulations usually have to treat very large numbers of particles which requires highly specialized solution schemes and accordingly powerful computers. Thus, it is beneficial to keep these computations separated from the FE simulation and to set up a suitable information transfer between the respective domains. Several options to achieve this decoupling are developed in the following, their qualitative and quantitative implications are discussed in detail in Section 5 while Section 6 is concluding on appropriate ansatzes for a staggered solution.

Four groups (I-IV) of staggered schemes will be introduced and investigated in the subsequent sections:

I "horizontal" decomposition:

FE-, particle- and Lagrange multiplier domains are each treated separately

II "vertical" decomposition of particle domain: particles in bridging domain and pure particle domain are each treated separately

III "vertical" decomposition of FE domain: pure FE part and bridging FE part are each treated separately

IV combines II and III.

\subsection{Staggered Computation I}

Recalling the one dimensional system used above, Figure 6 shows different options of staggered computations, classified as group I. They share the property that each particular component of the solution $\mathbf{d}=\left[\mathbf{u}^{h} \mathbf{w} \boldsymbol{\lambda}^{h}\right]^{T}$ is computed separately from the other two.

Staggered Scheme Ia separates the computation of the particle displacements $\mathbf{w}$ from that of nodal displacements and Lagrange multipliers $\left[\mathbf{u}^{h} \boldsymbol{\lambda}^{h}\right]^{T}$. In contrast, in Staggered Scheme Ib the computation of the nodal displacements $\mathbf{u}^{h}$ is split off from the computation of particle displacements and Lagrange multipliers $\left[\begin{array}{ll}\mathbf{w} & \boldsymbol{\lambda}^{h}\end{array}\right]^{T}$. Finally, Staggered Scheme Ic isolates the computation of the Lagrange multipliers $\lambda^{h}$ from the computation of nodal and particle displacements $\left[\mathbf{u}^{h} \mathbf{w}\right]^{T}$.
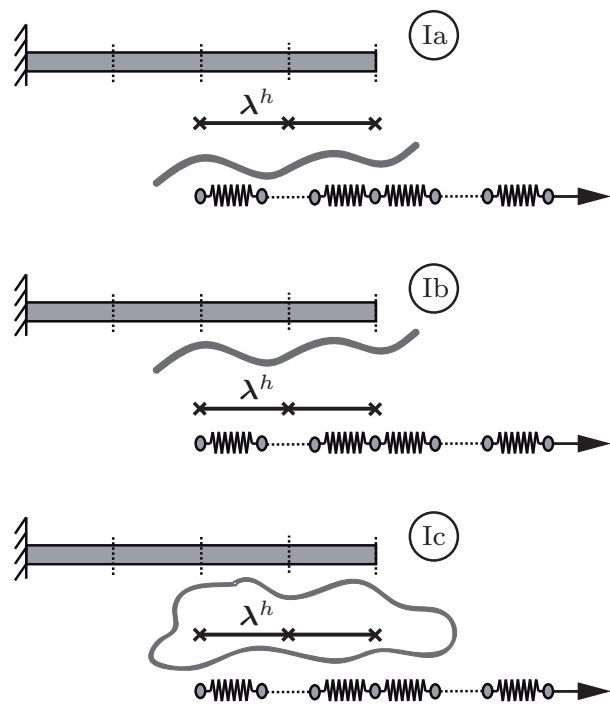

Fig. 6 Staggered computation I

\subsubsection{Staggered Scheme Ia}

Staggered Scheme Ia has been proposed by Zhang, Khare, Lu and Belytschko in [20], who also used a linear elastic formulation for the continuum. This scheme is sketched in Figure 7 and uses the following procedure:

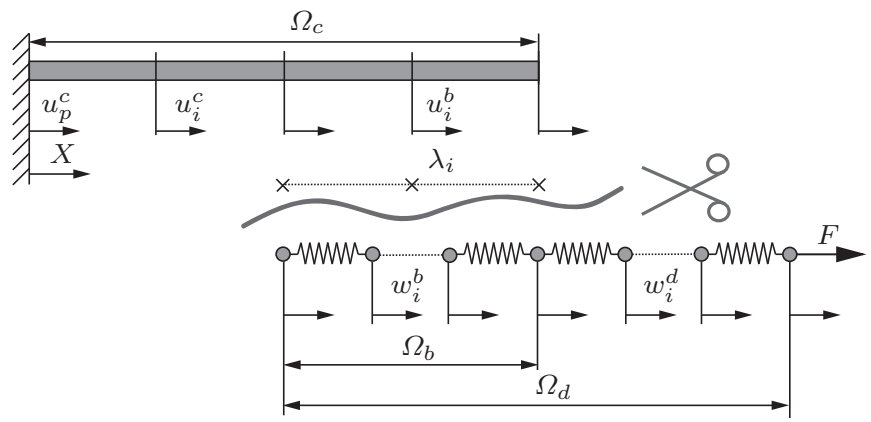

Fig. 7 Staggered Scheme Ia: FE domain $\Omega_{c}$ (nodal displacements $\mathbf{u}$ ) and Lagrange multipliers $\boldsymbol{\lambda}$ are separated from particle domain $\Omega_{d}$ (particle displacements $\mathbf{w}$ )

1. Guess initial particle displacements $\mathbf{w}_{0}$ and set: $\mathbf{w}_{n-1}=\mathbf{w}_{0}$; 
2. Solve the FE domain:

compute $\mathbf{u}_{n}^{h}$ and $\boldsymbol{\lambda}_{n}^{h}$ from

$\nabla_{\mathbf{u}^{h}, \boldsymbol{\lambda}^{h}} L\left(\mathbf{u}_{n}^{h}, \boldsymbol{\lambda}_{n}^{h} ; \mathbf{w}_{n-1}\right)=\mathbf{0}$,

which leads to the following system of equations:

$\mathbf{K}_{u \lambda} \cdot\left[\begin{array}{l}\mathbf{u}_{n}^{c} \\ \mathbf{u}_{n}^{b} \\ \boldsymbol{\lambda}_{n}^{h}\end{array}\right]=\left[\begin{array}{l}\mathbf{f}_{c}^{e x t} \\ \mathbf{f}_{b}^{e x t} \\ \mathbf{0}\end{array}\right]-\left[\begin{array}{l}\mathbf{K}_{c p}^{c} \\ \mathbf{K}_{b p}^{c} \\ \mathbf{0}\end{array}\right] \cdot \mathbf{u}_{p}^{c}-\left[\begin{array}{l}\mathbf{0} \\ \mathbf{0} \\ \mathbf{G}_{\lambda d}\end{array}\right] \cdot \mathbf{w}_{n-1}^{b}$

with $\quad \mathbf{K}_{u \lambda}=\left[\begin{array}{lll}\mathbf{K}_{c c}^{c} & \mathbf{K}_{c b}^{c} & \mathbf{0} \\ \mathbf{K}_{b c}^{c} & \mathbf{K}_{b b}^{c} & \mathbf{G}_{c \lambda} \\ \mathbf{0} & \mathbf{G}_{\lambda c} & \mathbf{0}\end{array}\right]$

3. Equilibrate the particle domain:

compute $\mathbf{w}_{n}$ from $L\left(\mathbf{w}_{n} ; \mathbf{u}_{n}^{h}, \boldsymbol{\lambda}_{n}^{h}\right) \rightarrow$ min with

$$
\begin{aligned}
L & =\frac{1}{2} \mathbf{u}^{h T} \cdot \mathbf{K}^{c} \cdot \mathbf{u}^{h}+\frac{1}{2} \mathbf{w}^{T} \cdot \mathbf{K}^{d} \cdot \mathbf{w} \\
& +\mathbf{u}^{b T} \cdot \mathbf{G}_{c \lambda} \cdot \boldsymbol{\lambda}^{h}+\mathbf{w}^{b T} \cdot \mathbf{G}_{d \lambda} \cdot \boldsymbol{\lambda}^{h} \\
& -\mathbf{u}^{h T} \cdot\left[\begin{array}{lll}
\mathbf{f}_{u}^{\text {ext }} & \mathbf{f}_{c}^{\text {ext }} & \mathbf{f}_{b}^{\text {ext }}
\end{array}\right]^{T} \\
& -\mathbf{w}^{T} \cdot\left[\begin{array}{ll}
\mathbf{g}_{b}^{\text {ext }} & \mathbf{g}_{d}^{\text {ext }}
\end{array}\right]^{T}
\end{aligned}
$$

and the first derivative with respect to $\mathbf{w}$

$\nabla_{\mathbf{w}} L=\mathbf{K}^{d} \cdot \mathbf{w}+\left[\begin{array}{l}\mathbf{G}_{d \lambda} \\ \mathbf{0}\end{array}\right] \cdot \lambda^{h}-\left[\begin{array}{l}\mathbf{g}_{b}^{e x t} \\ \mathbf{g}_{d}^{\text {ext }}\end{array}\right]$

The second derivative of $L$ with respect to $\mathbf{w}$

$\nabla_{\mathbf{w}}^{2} L=\mathbf{K}^{d}$

is not invertible since the particle domain is free of any DiRichlet boundary conditions. Thus, a minimum of (31) cannot be computed by minimizers which require the inverse second derivative of the problem, i.e. all Newton methods are discarded and substituted by a BFGS minimizer. We here employed the Matlab-based toolbox "minFunc" provided by [16] to obtain a minimum of (56) with respect to $\mathbf{w}$. All numerical examples have been computed by using only one BFGS update in each staggered iteration step $n$ which does not require the inverse of the second derivative (57). Instead, $\mathbf{w}_{n}$ is obtained as a function of the first derivative of the previous step $\nabla_{\mathbf{w}} L\left(\mathbf{w}_{\mathbf{n}-\mathbf{1}}\right)$ and of a step length $t$ which is computed within the minFunc toolbox using a Wolfe line search algorithm.
The advantage of this scheme is a clean separation of the computations in the different domains. Unlike to the monolithic case, a certain number of iteration steps between both domains is necessary and leads to more expensive computations.

\subsubsection{Staggered Scheme Ib}

Staggered Scheme Ib separates the computation in the FE domain from the computation of the Lagrange multipliers and the particle displacements. Although this scheme does not provide convergence in our experience, its algorithm is given for the sake of completeness:

1. Guess initial nodal displacements $\mathbf{u}_{0}^{h}$ and set: $\mathbf{u}_{n-1}^{h}=\mathbf{u}_{0}^{h}$;

2. Solve the particle domain $\mathbf{w}_{n}, \boldsymbol{\lambda}_{n}^{h}$ from $\nabla L_{\mathbf{w}, \boldsymbol{\lambda}^{h}}\left(\mathbf{w}_{n}, \boldsymbol{\lambda}_{n}^{h} ; \mathbf{u}_{n-1}^{h}\right)=\mathbf{0}:$

$$
\begin{aligned}
& \mathbf{K}_{w \lambda} \cdot\left[\begin{array}{l}
\mathbf{w}_{n}^{b} \\
\mathbf{w}_{n}^{d} \\
\boldsymbol{\lambda}_{n}^{h}
\end{array}\right]=\left[\begin{array}{l}
\mathbf{g}_{b}^{e x t} \\
\mathbf{g}_{d}^{e x t} \\
\mathbf{0}
\end{array}\right]-\left[\begin{array}{l}
\mathbf{0} \\
\mathbf{0} \\
\mathbf{G}_{\lambda c}
\end{array}\right] \cdot \mathbf{u}_{n-1}^{b} \\
& \text { with } \quad \mathbf{K}_{w \lambda}=\left[\begin{array}{lll}
\mathbf{K}_{b b}^{d} & \mathbf{K}_{b d}^{d} & \mathbf{G}_{d \lambda} \\
\mathbf{K}_{d b}^{d} & \mathbf{K}_{d d}^{d} & \mathbf{0} \\
\mathbf{G}_{\lambda d} & \mathbf{0} & \mathbf{0}
\end{array}\right] \text {; }
\end{aligned}
$$

3. Solve the FE domain

$\mathbf{u}_{n}^{h}$ from $\nabla_{\mathbf{u}^{h}} L\left(\mathbf{u}_{n}^{h} ; \mathbf{w}_{n}, \boldsymbol{\lambda}_{n}^{h}\right)=\mathbf{0}:$

$$
\mathbf{K}_{r e d}^{c} \cdot\left[\begin{array}{l}
\mathbf{u}_{n}^{c} \\
\mathbf{u}_{n}^{b}
\end{array}\right]=\left[\begin{array}{l}
\mathbf{f}_{c}^{e x t} \\
\mathbf{f}_{b}^{e x t}
\end{array}\right]-\left[\begin{array}{l}
\mathbf{K}_{c p}^{c} \\
\mathbf{K}_{b p}^{c}
\end{array}\right] \cdot \mathbf{u}_{p}^{c}-\left[\begin{array}{l}
\mathbf{0} \\
\mathbf{G}_{c \lambda}
\end{array}\right] \cdot \boldsymbol{\lambda}_{n}^{h}
$$

with $\quad \mathbf{K}_{r e d}^{c}=\left[\begin{array}{ll}\mathbf{K}_{c c}^{c} & \mathbf{K}_{c b}^{c} \\ \mathbf{K}_{b c}^{c} & \mathbf{K}_{b b}^{c}\end{array}\right]$

4. Return to 2. with $\mathbf{u}_{n}^{h}$ fixed.

\subsubsection{Staggered Scheme Ic}

In Staggered Scheme Ic (cf. Figure 8) the computation of the Lagrange multipliers is separated from both the computation of the nodal and the particle displacements. The following iteration procedure was used:

1. Guess initial Lagrange multipliers $\boldsymbol{\lambda}_{0}^{h}$ and set: $\boldsymbol{\lambda}_{n-1}^{h}=\boldsymbol{\lambda}_{0}^{h}$;

4. Return to 2 . with $\mathbf{w}_{n}$ fixed. 


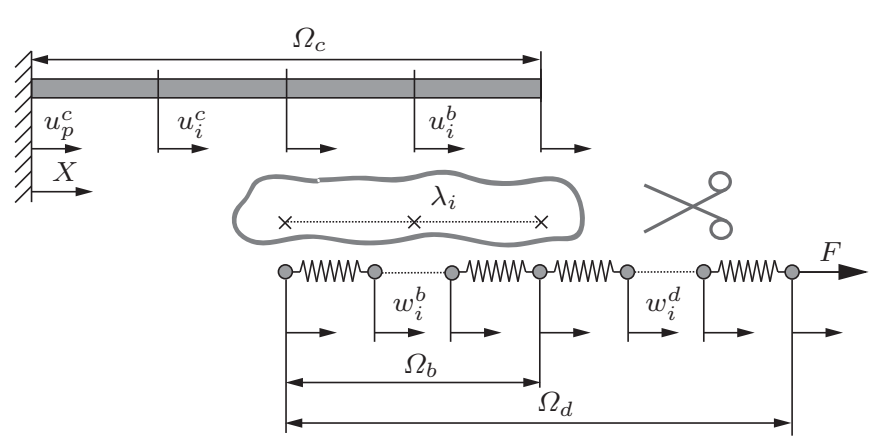

Fig. 8 Staggered Scheme Ic: separation of Lagrange multipliers

2. Iterate FE domain and particle domain until equilibrium:

$\mathbf{u}_{n}^{h}, \mathbf{w}_{n}$ from $L\left(\mathbf{u}_{n}^{h}, \mathbf{w}_{n} ; \boldsymbol{\lambda}_{n-1}^{h}\right) \rightarrow \min$.

The first derivative of $L$ with respect to $\mathbf{u}^{h}$ and $\mathbf{w}$ can be written as

$\nabla_{\mathbf{u}^{h}, \mathbf{w}} L=$

$\mathbf{K}_{u w} \cdot\left[\begin{array}{l}\mathbf{u}^{c} \\ \mathbf{u}^{b} \\ \mathbf{w}^{b} \\ \mathbf{w}^{d}\end{array}\right]+\left[\begin{array}{l}\mathbf{0} \\ \mathbf{G}_{c \lambda} \\ \mathbf{G}_{d \lambda} \\ \mathbf{0}\end{array}\right] \cdot \boldsymbol{\lambda}^{h}+\left[\begin{array}{l}\mathbf{K}_{c p}^{c} \\ \mathbf{K}_{b p}^{c} \\ \mathbf{0} \\ \mathbf{0}\end{array}\right] \cdot \mathbf{u}_{p}^{c}-\left[\begin{array}{l}\mathbf{f}_{c}^{e x t} \\ \mathbf{f}_{b}^{e x t} \\ \mathbf{g}_{b}^{e x t} \\ \mathbf{g}_{d}^{e x t}\end{array}\right]$

with $\quad \mathbf{K}_{u w}=\left[\begin{array}{cccc}\mathbf{K}_{c c}^{c} & \mathbf{K}_{c b}^{c} & \mathbf{0} & \mathbf{0} \\ \mathbf{K}_{b c}^{c} & \mathbf{K}_{b b}^{c} & \mathbf{0} & \mathbf{0} \\ \mathbf{0} & \mathbf{0} & \mathbf{K}_{b b}^{d} & \mathbf{K}_{b d}^{d} \\ \mathbf{0} & \mathbf{0} & \mathbf{K}_{d b}^{d} & \mathbf{K}_{d d}^{d}\end{array}\right]$.

For the investigated system a not invertible second derivative of $L$ with respect to $\mathbf{u}^{h}$ and $\mathbf{w}$

$\nabla_{\mathbf{u}^{h}, \mathbf{w}}^{2} L=\mathbf{K}_{u w}$

is obtained, i.e. equation (31) has to be solved again by using a BFGS minimizer.

3. Compute the Lagrange multipliers:

$\boldsymbol{\lambda}_{n}^{h}$ from $\nabla_{\mathbf{u}^{b}, \mathbf{w}^{b}} L\left(\boldsymbol{\lambda}_{n}^{h} ; \mathbf{u}_{n}^{h}, \mathbf{w}_{n}\right)=\mathbf{0}:$

$\mathbf{G}_{\lambda} \cdot \boldsymbol{\lambda}_{n}^{h}=\left[\begin{array}{c}\mathbf{f}_{b}^{e x t} \\ \mathbf{g}_{b}^{\text {ext }}\end{array}\right]-\left[\begin{array}{ccccc}\mathbf{K}_{b p}^{c} & \mathbf{K}_{b c}^{c} & \mathbf{K}_{b b}^{c} & \mathbf{0} & \mathbf{0} \\ \mathbf{0} & \mathbf{0} & \mathbf{0} & \mathbf{K}_{b b}^{d} & \mathbf{K}_{b d}^{d}\end{array}\right] \cdot\left[\begin{array}{c}\mathbf{u}_{n}^{h} \\ \mathbf{w}_{n}\end{array}\right]$

with $\quad \mathbf{G}_{\lambda}=\left[\begin{array}{l}\mathbf{G}_{c \lambda} \\ \mathbf{G}_{d \lambda}\end{array}\right]$.

Since $\mathbf{G}_{\lambda}$ is not a square matrix, both sides of (66) have to be multiplied from the left hand side by $\mathbf{G}_{\lambda}^{T}$ and solved for $\boldsymbol{\lambda}_{n}^{h}$. However, it has to be remarked, that $\boldsymbol{\lambda}_{n}^{h}$ is not an exact solution for the overdetermined system (66). Furthermore, (66) is related to rows 3 and 4 in system (49) and the dimension of $\boldsymbol{\lambda}^{h}$ corresponds to the number of Lagrange multipliers.

\section{Return to 2. with $\boldsymbol{\lambda}_{n}^{h}$ fixed.}

In contrast to Staggered Scheme Ia, the computations in the FE domain and the particle domain are not separated from each other. It turns out that the number of iteration loops is even slightly higher than for Staggered Scheme Ia, see below.

It has to be remarked that all schemes of this group suffer from the fact that the particle domain is free of DiRichlet boundary conditions, i.e. the stiffness matrix $\mathbf{K}^{d}$ is singular. Thus, a minimization tool has been employed here which does not necessitate to compute the inverse of $\mathbf{K}^{d}$. Another option to overcome this problem has been described in [8], where discrete mixed Arlequin problems were solved using a preconditioned FETI-like solver. Similar to our contribution, the computations of particle/continuum displacements and Lagrange multipliers are separated from each other. Instead of employing a minimization tool, the pseudoinverse of a singular matrix is used together with a certain elimination of rigid body modes. A detailed investigation of the FETI ("Finite Element Tearing and Interconnecting“) solver can be found in [11].

\subsection{Staggered Computation II}

Further options of staggered computations are shown in Figure 9. In both schemes the particle domain is divided into two parts, thus the particle displacements in the bridging domain are decoupled from those in the pure particle domain.

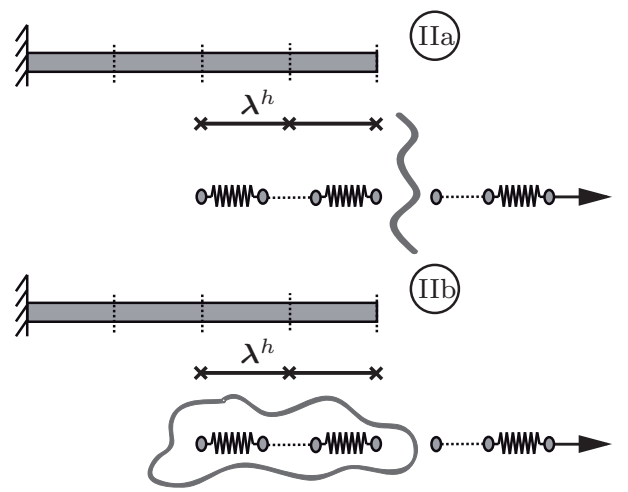

Fig. 9 Staggered computation II 
Staggered Scheme IIa separates the computation of the displacements in the pure particle domain $\mathbf{w}^{d}$ from the computation of nodal displacements, particle displacements in the bridging domain and Lagrange multipliers $\left[\mathbf{u}^{h} \mathbf{w}^{b} \boldsymbol{\lambda}^{h}\right]^{T}$.

In contrast, Staggered Scheme IIb isolates the computation of the particle displacements in the bridging domain $\mathbf{w}^{b}$ from the computation of nodal displacements, particle displacements in the pure particle domain and Lagrange multipliers $\left[\mathbf{u}^{h} \mathbf{w}^{d} \boldsymbol{\lambda}^{h}\right]^{T}$.

\subsubsection{Staggered Scheme IIa}

The following algorithm is used for Staggered Scheme IIa:

1. Guess initial particle displacements in the pure particle domain $\mathbf{w}_{0}^{d}$

and set: $\mathbf{w}_{n-1}^{d}=\mathbf{w}_{0}^{d}$;

2. Compute all nodal displacements as well as the particle displacements in the bridging domain:

$\mathbf{u}_{n}^{h}, \boldsymbol{\lambda}_{n}^{h}, \mathbf{w}_{n}^{b}$ from

$\nabla L_{\mathbf{u}^{h}, \mathbf{w}^{b}, \boldsymbol{\lambda}^{h}}\left(\mathbf{u}_{n}^{h}, \mathbf{w}_{n}^{b}, \boldsymbol{\lambda}_{n}^{h} ; \mathbf{w}_{n-1}^{d}\right)=\mathbf{0}:$

$\mathbf{K}_{u w \lambda}^{a} \cdot\left[\begin{array}{l}\mathbf{u}_{n}^{c} \\ \mathbf{u}_{n}^{b} \\ \mathbf{w}_{n}^{b} \\ \boldsymbol{\lambda}_{n}^{h}\end{array}\right]=\left[\begin{array}{l}\mathbf{f}_{c}^{e x t} \\ \mathbf{f}_{b}^{e x t} \\ \mathbf{g}_{b}^{e x t} \\ \mathbf{0}\end{array}\right]-\left[\begin{array}{l}\mathbf{K}_{c p}^{c} \\ \mathbf{K}_{b p}^{c} \\ \mathbf{0} \\ \mathbf{0}\end{array}\right] \cdot \mathbf{u}_{p}^{c}-\left[\begin{array}{l}\mathbf{0} \\ \mathbf{0} \\ \mathbf{K}_{b d}^{d} \\ \mathbf{0}\end{array}\right] \cdot \mathbf{w}_{n-1}^{d}$

with $\quad \mathbf{K}_{u w \lambda}^{a}=\left[\begin{array}{llll}\mathbf{K}_{c c}^{c} & \mathbf{K}_{c b}^{c} & \mathbf{0} & \mathbf{0} \\ \mathbf{K}_{b c}^{c} & \mathbf{K}_{b b}^{c} & \mathbf{0} & \mathbf{G}_{c \lambda} \\ \mathbf{0} & \mathbf{0} & \mathbf{K}_{b b}^{d} & \mathbf{G}_{d \lambda} \\ \mathbf{0} & \mathbf{G}_{\lambda c} & \mathbf{G}_{\lambda d} & \mathbf{0}\end{array}\right]$

3. Solve the pure particle domain:

$\mathbf{w}_{n}^{d}$ from $\nabla L_{\mathbf{w}^{d}}\left(\mathbf{w}_{n}^{d} ; \mathbf{u}_{n}^{h}, \mathbf{w}_{n}^{b}, \boldsymbol{\lambda}_{n}^{h}\right)=\mathbf{0}:$

$\mathbf{K}_{d d}^{d} \cdot \mathbf{w}_{n}^{d}=\mathbf{g}_{d}^{e x t}-\mathbf{K}_{d b}^{d} \cdot \mathbf{w}_{n}^{b}$

4. return to 2 . with $\mathbf{w}_{n}^{d}$ fixed.

In Figure 10, Staggered Scheme IIa is sketched. It is obvious, that this scheme does not separate FE and particle domain as far as Staggered Scheme Ia, but it requires much fewer iteration steps. When considering very simple toy systems, as it is done in Section 5 , this is not surprising: actually only one displacement, namely that of the particle between the two "domains", has to

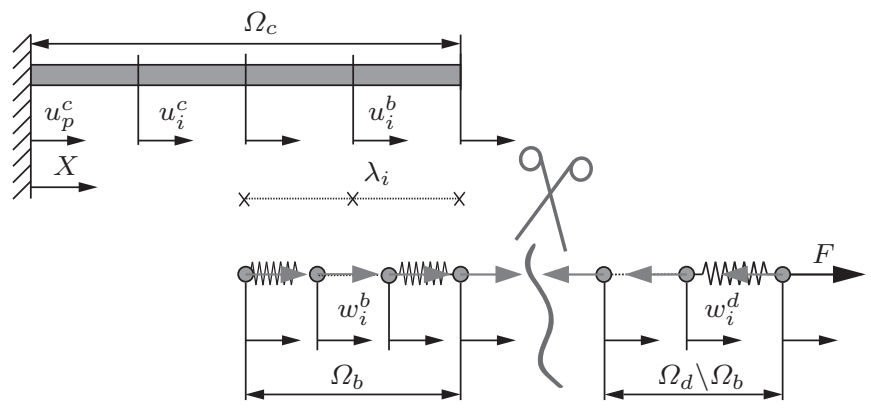

Fig. 10 Staggered computation IIa: separation of pure particle domain $\Omega_{d}$ from bridging domain $\Omega_{b}$ and $\mathrm{FE}$ domain $\Omega_{c}$; gray arrows: section forces due to domain decomposition

be exchanged in each iteration step. Please note, however, that this observation cannot be transferred easily to more complex, three-dimensional systems. Instead, a detailed study would be necessary.

\subsubsection{Staggered Scheme IIb}

As a variation of Staggered Scheme IIa, the following algorithm was investigated:

1. Guess initial particle displacements in the bridging domain $\mathbf{w}_{0}^{b}$

and set: $\mathbf{w}_{n-1}^{b}=\mathbf{w}_{0}^{b}$;

2. Solve the FE domain and the pure particle domain: $\mathbf{u}_{n}^{h}, \boldsymbol{\lambda}_{n}^{h}, \mathbf{w}_{n}^{d}$ from $\nabla L_{\mathbf{u}^{h}, \mathbf{w}^{d}, \boldsymbol{\lambda}^{h}}\left(\mathbf{u}_{n}^{h}, \mathbf{w}_{n}^{d}, \boldsymbol{\lambda}_{n}^{h} ; \mathbf{w}_{n-1}^{b}\right)=\mathbf{0}:$

$\mathbf{K}_{u w \lambda}^{b} \cdot\left[\begin{array}{c}\mathbf{u}_{n}^{c} \\ \mathbf{u}_{n}^{b} \\ \mathbf{w}_{n}^{d} \\ \boldsymbol{\lambda}_{n}^{h}\end{array}\right]=\left[\begin{array}{l}\mathbf{f}_{c}^{e x t} \\ \mathbf{f}_{b}^{e x t} \\ \mathbf{g}_{d}^{e x t} \\ \mathbf{0}\end{array}\right]-\left[\begin{array}{l}\mathbf{K}_{c p}^{c} \\ \mathbf{K}_{b p}^{c} \\ \mathbf{0} \\ \mathbf{0}\end{array}\right] \cdot \mathbf{u}_{p}^{c}-\left[\begin{array}{l}\mathbf{0} \\ \mathbf{0} \\ \mathbf{K}_{d b}^{d} \\ \mathbf{G}_{\lambda d}\end{array}\right] \cdot \mathbf{w}_{n-1}^{b}$

with $\quad \mathbf{K}_{u w \lambda}^{b}=\left[\begin{array}{llll}\mathbf{K}_{c c}^{c} & \mathbf{K}_{c b}^{c} & \mathbf{0} & \mathbf{0} \\ \mathbf{K}_{b c}^{c} & \mathbf{K}_{b b}^{c} & \mathbf{0} & \mathbf{G}_{c \lambda} \\ \mathbf{0} & \mathbf{0} & \mathbf{K}_{d d}^{d} & \mathbf{0} \\ \mathbf{0} & \mathbf{G}_{\lambda c} & \mathbf{0} & \mathbf{0}\end{array}\right]$

3. Calculate particle displacements in the bridging domain:

$$
\begin{aligned}
& \mathbf{w}_{n}^{b} \text { from } \nabla L_{\mathbf{w}^{b}}\left(\mathbf{w}_{n}^{b} ; \mathbf{u}_{n}^{h}, \mathbf{w}_{n}^{d}, \boldsymbol{\lambda}_{n}^{h}\right)=\mathbf{0}: \\
& \mathbf{K}_{b b}^{d} \cdot \mathbf{w}_{n}^{b}=\mathbf{g}_{b}^{e x t}-\mathbf{K}_{b d}^{d} \cdot \mathbf{w}_{n}^{d}-\mathbf{G}_{d \lambda} \cdot \boldsymbol{\lambda}_{n}^{h} ;
\end{aligned}
$$

4. return to 2 . with $\mathbf{w}_{n}^{b}$ fixed. 
Unfortunately, in our test calculations this scheme does not converge to a solution. Furthermore, this separation of computations does not really decouple FE and MD. Thus, it is not necessary to continue with further investigations here.

\subsection{Staggered Computation III}

Instead of dividing the particle domain, the FE domain can be dissected into two parts as shown in Figure 11.

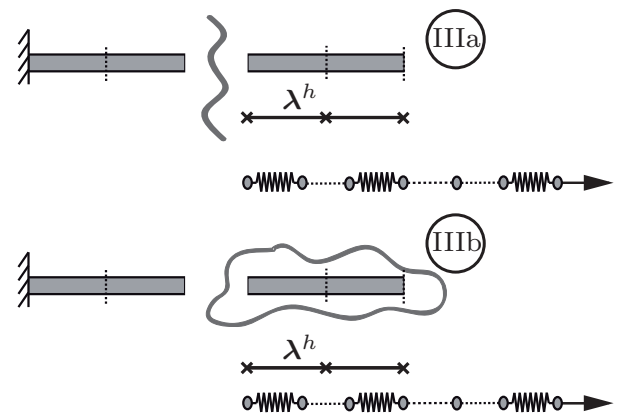

Fig. 11 Staggered computation III

In Staggered Scheme IIIa, the computation of the unknown nodal displacements in the pure FE domain $\mathbf{u}_{u}^{c}$ is split from that of nodal displacements in the bridging domain, particle displacements and Lagrange multipliers $\left[\mathbf{u}^{b} \mathbf{w} \boldsymbol{\lambda}^{h}\right]^{T}$.

Vice versa, Staggered Scheme IIIb isolates the computation of the nodal displacements in the bridging domain $\mathbf{u}^{b}$ from the computation of the unknown nodal displacements in the pure FE domain, the particle displacements and the Lagrange multipliers $\left[\mathbf{u}^{c} \mathbf{w} \boldsymbol{\lambda}^{h}\right]^{T}$.

\subsubsection{Staggered Scheme IIIa}

The following algorithm realizes Staggered Scheme IIIa:

1. Guess initial nodal displacements in the pure FE domain $\mathbf{u}_{0}^{c}$

and set: $\mathbf{u}_{n-1}^{c}=\mathbf{u}_{0}^{c}$;

2. Solve the FE part of the bridging domain and the whole particle domain:

$\mathbf{u}_{n}^{b}, \mathbf{w}_{n}, \boldsymbol{\lambda}_{n}^{h}$ from

$$
\nabla L_{\mathbf{u}^{b}, \mathbf{w}, \boldsymbol{\lambda}^{h}}\left(\mathbf{u}_{n}^{b}, \mathbf{w}_{n}, \boldsymbol{\lambda}_{n}^{h} ; \mathbf{u}_{n-1}^{c}\right)=\mathbf{0}
$$

$\mathbf{K}_{u w \lambda}^{c} \cdot\left[\begin{array}{l}\mathbf{u}_{n}^{b} \\ \mathbf{w}_{n}^{b} \\ \mathbf{w}_{n}^{d} \\ \boldsymbol{\lambda}_{n}^{h}\end{array}\right]=\left[\begin{array}{l}\mathbf{f}_{c}^{e x t} \\ \mathbf{g}_{b}^{e x t} \\ \mathbf{g}_{d}^{e x t} \\ \mathbf{0}\end{array}\right]-\left[\begin{array}{l}\mathbf{K}_{b p}^{c} \\ \mathbf{0} \\ \mathbf{0} \\ \mathbf{0}\end{array}\right] \cdot \mathbf{u}_{p}^{c}-\left[\begin{array}{l}\mathbf{K}_{b c}^{c} \\ \mathbf{0} \\ \mathbf{0} \\ \mathbf{0}\end{array}\right] \cdot \mathbf{u}_{n-1}^{c}$

with $\quad \mathbf{K}_{u w \lambda}^{c}=\left[\begin{array}{llll}\mathbf{K}_{b b}^{c} & \mathbf{0} & \mathbf{0} & \mathbf{G}_{c \lambda} \\ \mathbf{0} & \mathbf{K}_{b b}^{d} & \mathbf{K}_{b d}^{d} & \mathbf{G}_{d \lambda} \\ \mathbf{0} & \mathbf{K}_{d b}^{d} & \mathbf{K}_{d d}^{d} & \mathbf{0} \\ \mathbf{G}_{\lambda c} & \mathbf{G}_{\lambda d} & \mathbf{0} & \mathbf{0}\end{array}\right] ;$

3. Solve the pure FE-domain:

$$
\begin{aligned}
& \mathbf{u}_{n}^{c} \text { from } \nabla L_{\mathbf{u}^{c}}\left(\mathbf{u}_{n}^{c} ; \mathbf{u}_{n}^{b}, \mathbf{w}_{n}, \boldsymbol{\lambda}_{n}^{h}\right)=\mathbf{0}: \\
& \mathbf{K}_{c c}^{c} \cdot \mathbf{u}_{n}^{c}=\mathbf{f}_{c}^{e x t}-\mathbf{K}_{c p}^{c} \cdot \mathbf{u}_{p}^{c}-\mathbf{K}_{c b}^{c} \cdot \mathbf{u}_{n}^{b} ;
\end{aligned}
$$

4. Return to 2. with $\mathbf{u}_{n}^{c}$ fixed.

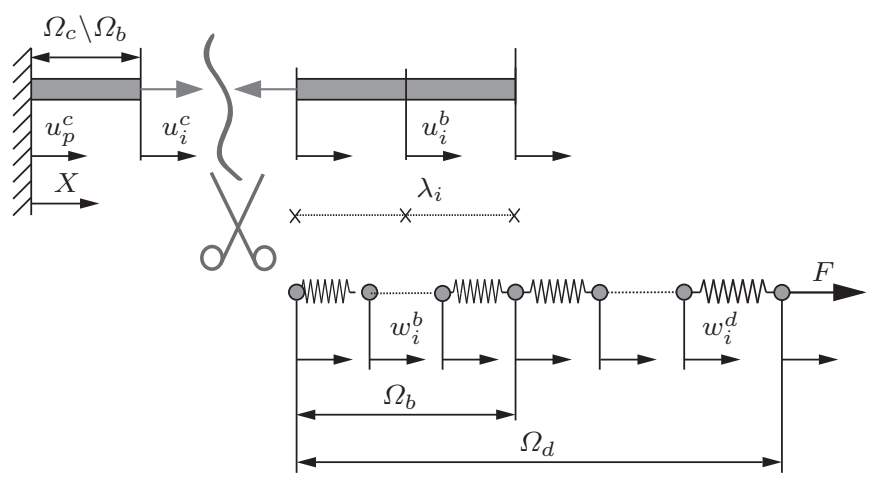

Fig. 12 Staggered computation IIIa, gray arrows: section forces due to domain decomposition

Figure 12 displays Staggered Scheme IIIa. Both domains are not separated as far as in Staggered Scheme Ia. However, it turned out that the necessary number of iterations between MD and FE is significantly lower, cf. the results section.

\subsubsection{Staggered Scheme IIIb}

In addition to Staggered Scheme IIIa, the following algorithm has been investigated:

1. Guess initial nodal displacements in the bridging domain $\mathbf{u}_{0}^{b}$

and set: $\mathbf{u}_{n-1}^{b}=\mathbf{u}_{0}^{b}$; 
2. Solve the pure FE domain and the whole particle domain:

$$
\begin{aligned}
& \mathbf{u}_{n}^{c}, \boldsymbol{\lambda}_{n}^{h}, \mathbf{w}_{n} \text { from } \\
& \nabla L_{\mathbf{u}^{c}, \mathbf{w}, \boldsymbol{\lambda}^{h}}\left(\mathbf{u}_{n}^{c}, \mathbf{w}_{n}, \boldsymbol{\lambda}_{n}^{h} ; \mathbf{u}_{n-1}^{b}\right)=\mathbf{0}:
\end{aligned}
$$$$
\mathbf{K}_{u w \lambda}^{d} \cdot\left[\begin{array}{c}
\mathbf{u}_{n}^{c} \\
\mathbf{w}_{n}^{b} \\
\mathbf{w}_{n}^{d} \\
\boldsymbol{\lambda}_{n}^{h}
\end{array}\right]=\left[\begin{array}{l}
\mathbf{f}_{c}^{e x t} \\
\mathbf{g}_{b}^{\text {ext }} \\
\mathbf{g}_{d}^{\text {ext }} \\
\mathbf{0}
\end{array}\right]-\left[\begin{array}{l}
\mathbf{K}_{c p}^{c} \\
\mathbf{0} \\
\mathbf{0} \\
\mathbf{0}
\end{array}\right] \cdot \mathbf{u}_{p}^{c}-\left[\begin{array}{l}
\mathbf{K}_{c b}^{c} \\
\mathbf{0} \\
\mathbf{0} \\
\mathbf{G}_{\lambda c}
\end{array}\right] \cdot \mathbf{u}_{n-1}^{b}
$$

$$
\text { with } \quad \mathbf{K}_{u w \lambda}^{d}=\left[\begin{array}{llll}
\mathbf{K}_{c c}^{c} & \mathbf{0} & \mathbf{0} & \mathbf{0} \\
\mathbf{0} & \mathbf{K}_{b b}^{d} & \mathbf{K}_{b d}^{d} & \mathbf{G}_{d \lambda} \\
\mathbf{0} & \mathbf{K}_{d b}^{d} & \mathbf{K}_{d d}^{d} & \mathbf{0} \\
\mathbf{0} & \mathbf{G}_{\lambda d} & \mathbf{0} & \mathbf{0}
\end{array}\right]
$$

3. Compute the nodal displacements in the bridging domain:

$\mathbf{u}_{n}^{b}$ from $\nabla L_{\mathbf{u}^{b}}\left(\mathbf{u}_{n}^{b} ; \mathbf{w}_{n}, \boldsymbol{\lambda}_{n}^{h}\right)=\mathbf{0}:$

$$
\mathbf{K}_{b b}^{c} \cdot \mathbf{u}_{n}^{b}=\mathbf{f}_{b}^{e x t}-\mathbf{K}_{b p}^{c} \cdot \mathbf{u}_{p}^{c}-\mathbf{K}_{b c}^{c} \cdot \mathbf{u}_{n}^{c}-\mathbf{G}_{c \lambda} \cdot \boldsymbol{\lambda}_{n}^{h}
$$

4. Return to 2. with $\mathbf{u}_{n}^{b}$ fixed.

Like Staggered Scheme IIb, this scheme does not converge in our experience, i.e. further investigations can be omitted.

\subsection{Staggered Computation IV}

Staggered Schemes IIa and IIIa can be combined to Staggered Scheme IV which is structured as follows: Computation of the Lagrange multipliers, nodal and particle displacements in the bridging domain $\left[\mathbf{u}^{b} \mathbf{w}^{b} \boldsymbol{\lambda}^{h}\right]^{T}$, computation of the unknown nodal displacements in the pure $\mathrm{FE}$ domain $\mathbf{u}_{u}^{c}$, and, finally, computation of the particle displacements in the pure particle domain $\mathbf{w}^{d}$, cf. Figure 13.

The following algorithm is used for Staggered Scheme IV:

1. Guess initial nodal and particle displacements in the pure $\mathrm{FE}$ domain $\mathbf{u}_{0}^{c}$ and particle domain $\mathbf{w}_{0}^{d}$, respectively,

and set: $\mathbf{u}_{n-1}^{c}=\mathbf{u}_{0}^{c}, \mathbf{w}_{n-1}^{d}=\mathbf{w}_{0}^{d}$;
2. Solve the bridging domain:

$$
\begin{aligned}
& \mathbf{u}_{n}^{b}, \mathbf{w}_{n}^{b}, \boldsymbol{\lambda}_{n}^{h} \text { from } \\
& \nabla L_{\mathbf{u}^{b}, \mathbf{w}^{b}, \boldsymbol{\lambda}^{h}}\left(\mathbf{u}_{n}^{b}, \mathbf{w}_{n}^{b}, \boldsymbol{\lambda}_{n}^{h} ; \mathbf{u}_{n-1}^{c}, \mathbf{w}_{n-1}^{d}\right)=\mathbf{0}:
\end{aligned}
$$

$$
\mathbf{K}_{u w \lambda}^{e} \cdot\left[\begin{array}{l}
\mathbf{u}_{n}^{b} \\
\mathbf{w}_{n}^{b} \\
\boldsymbol{\lambda}_{n}^{h}
\end{array}\right]=\left[\begin{array}{l}
\mathbf{f}_{b}^{e x t} \\
\mathbf{g}_{b}^{e x t} \\
\mathbf{0}
\end{array}\right]-\left[\begin{array}{lll}
\mathbf{K}_{b p}^{c} & \mathbf{K}_{b c}^{c} \mathbf{0} \\
\mathbf{0} & \mathbf{0} & \mathbf{K}_{b d}^{d} \\
\mathbf{0} & \mathbf{0} & \mathbf{0}
\end{array}\right] \cdot\left[\begin{array}{l}
\mathbf{u}_{p}^{c} \\
\mathbf{u}_{n-1}^{c} \\
\mathbf{w}_{n-1}^{d}
\end{array}\right]
$$

$$
\text { with } \quad \mathbf{K}_{u w \lambda}^{e}=\left[\begin{array}{lll}
\mathbf{K}_{b b}^{c} & \mathbf{0} & \mathbf{G}_{c \lambda} \\
\mathbf{0} & \mathbf{K}_{b b}^{d} & \mathbf{G}_{d \lambda} \\
\mathbf{G}_{\lambda c} & \mathbf{G}_{\lambda d} & \mathbf{0}
\end{array}\right]
$$

3. Solve the pure FE domain:

$$
\begin{aligned}
& \mathbf{u}_{n}^{c} \text { from } \nabla L_{\mathbf{u}^{c}}\left(\mathbf{u}_{n}^{c} ; \mathbf{u}_{n}^{b}, \mathbf{w}_{n}, \boldsymbol{\lambda}_{n}^{h}\right)=\mathbf{0}: \\
& \mathbf{K}_{c c}^{c} \cdot \mathbf{u}_{n}^{c}=\mathbf{f}_{c}^{e x t}-\mathbf{K}_{c p}^{c} \cdot \mathbf{u}_{p}^{c}-\mathbf{K}_{c b}^{c} \cdot \mathbf{u}_{n}^{b} ;
\end{aligned}
$$

4. Solve the pure particle domain:

$$
\mathbf{w}_{n}^{d} \text { from } \nabla L_{\mathbf{w}^{d}}\left(\mathbf{w}_{n}^{d} ; \mathbf{u}_{n}^{h}, \mathbf{w}_{n}^{b}, \boldsymbol{\lambda}_{n}^{h}\right)=\mathbf{0}:
$$

$\mathbf{K}_{d d}^{d} \cdot \mathbf{w}_{n}^{d}=\mathbf{g}_{d}^{e x t}-\mathbf{K}_{d b}^{d} \cdot \mathbf{w}_{n}^{b} ;$

5. Return to 2. with $\mathbf{u}_{n}^{c}$ and $\mathbf{w}_{n}^{d}$ fixed.

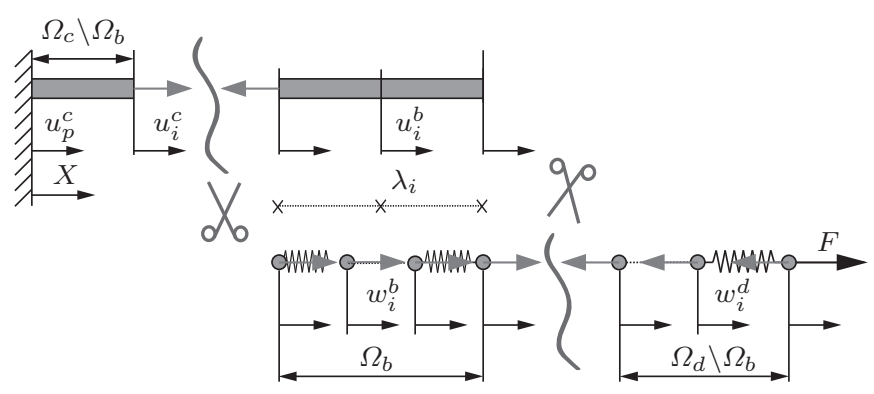

Fig. 13 Staggered computation IV, gray arrows: section forces due to domain decompositions

Figure 13 sketches Staggered Scheme IV which combines Staggered Schemes IIa and IIIa. Numerical examples in one dimension show that the number of iterations until equilibrium is slightly higher than in Staggered Scheme IIa, but significantly lower than in Staggered Scheme Ia. Thus, this scheme seems to be an appropriate candidate for staggered computations on different CPUs. 
Table 1 Qualitative evaluation of criteria: very good $(+++)$, $\operatorname{good}(++)$, okay $(+)$, bad $(-)$

\begin{tabular}{|c|c|c|c|}
\hline Scheme & $\begin{array}{c}\text { Need for } \\
\text { first order } \\
\text { methods }\end{array}$ & $\begin{array}{c}\text { Convergence } \\
\text { behavior }\end{array}$ & $\begin{array}{c}\text { Degree of } \\
\text { decoupling }\end{array}$ \\
\hline Ia & yes & - & +++ \\
\hline Ib & no & no convergence & ++ \\
\hline Ic & yes & - & ++ \\
\hline IIa & no & + & ++ \\
\hline IIb & no & no convergence & + \\
\hline IIIa & no & +++ & + \\
\hline IIIb & no & no convergence & + \\
\hline IV & no & + & ++ \\
\hline
\end{tabular}

\section{Qualitative and quantitative comparison, numerical examples}

First of all, the different staggered schemes have to be compared qualitatively. Therefore, three criteria are introduced and evaluated in Table 1 as follows:

- need for first order methods: denotes whether it is necessary to use first order solvers since second order methods (NEWTON methods) can not be used due to not invertible Hessians

- convergence behavior: qualitatively compares the number of iteration steps required

- degree of decoupling: evaluates to which extend computations in different domains are separated from each other

It is obvious that none of the staggering schemes tested can be considered as a perfect setup. While Staggered Scheme Ia separates the computations in the respective domains very well it shows only bad convergence. On the other hand, Staggered Scheme IIIa as a very fast converging scheme does not provide a complete separation of the particle domain from the $\mathrm{FE}$ domain as it would be desirable. We trace back the lack of convergence of Staggered Schemes Ib, IIb, and IIIb to a possible ill-posedness of the separate subproblems introduced by the decoupling of domains.

Beyond the qualitative comparison, a relative error of staggered computations with respect to the monolithic solution

$e=\frac{\left\|\mathbf{d}^{\text {stag }}-\mathbf{d}^{\text {mono }}\right\|_{2}}{\left\|\mathbf{d}^{\text {mono }}\right\|_{2}}$

is introduced and evaluated quantitatively, where $\mathbf{d}^{\text {mono }}$ and $\mathbf{d}^{\text {stag }}$ denote the solution vector $\left[\mathbf{u}^{h} \mathbf{w} \boldsymbol{\lambda}^{h}\right]^{T}$ in the monolithic and the staggered case, respectively, and $\mathbf{d}_{0}^{\text {stag }}=\mathbf{0}$ has been chosen as initial value.

Figure 14 displays the relative error $e$ as a function of the number of iterations $n$ between $\mathrm{FE}$ domain and particle region. As before, a toy system with only few degrees of freedom was chosen to ensure reasonable computing times: two finite elements in the pure continuum, two finite elements, two Lagrange multipliers and five particles in the bridging domain as well as four particles in the pure particle domain.

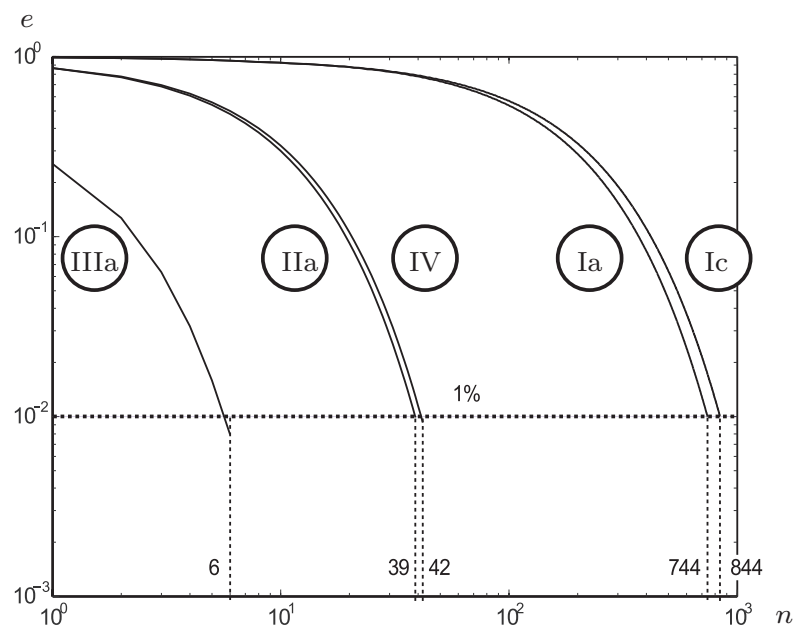

Fig. 14 Relative error $e$ vs. number of iterations $n, 5$ and 4 particles in bridging and pure particle domain, resp., $H^{1}$ norm in coupling constraint (29)

It is obvious that Staggered Schemes IIa and IV converge much faster than Staggered Schemes Ia and Ic. To achieve a relative error lower than $1 \%$ with respect to the monolithic solution, Staggered Scheme Ia requires 744 iteration loops whereas Staggered Scheme IIa requires only 39. However, Staggered Scheme IIIa converges even faster and needs only 6 iterations.

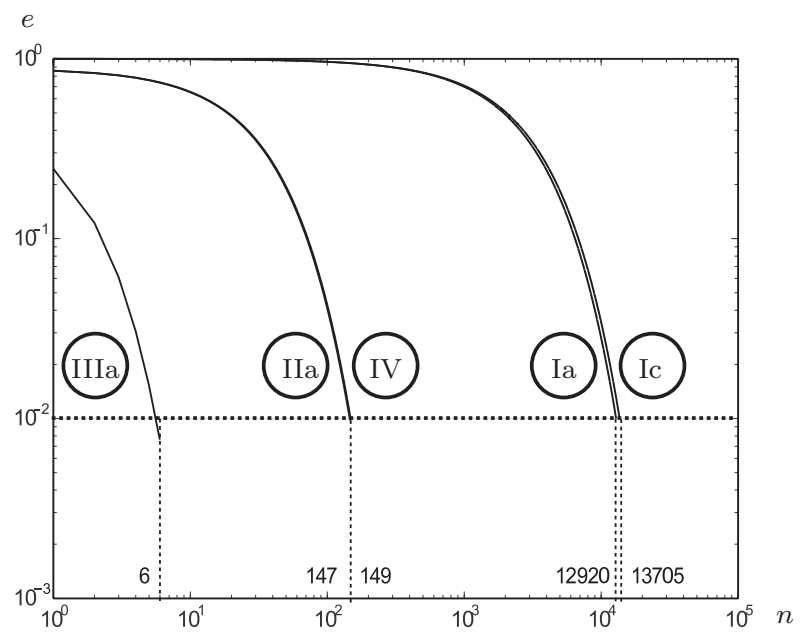

Fig. 15 Relative error $e$ vs. number of iterations $n, 17$ and 16 particles in bridging and pure particle domain, resp., $H^{1}$ norm in coupling constraint (29) 
Furthermore, an extended system with 17 particles in bridging and 16 particles in pure particle domain has been investigated to evaluate the influence of the size of the particle domain. Figure 15 depicts the results, which are qualitatively quite similar to those in Figure 14. The largest number of iterations is again required for Staggered Schemes Ia and Ic, whereas the number of iteration steps for Staggered Scheme IIIa does not increase at all.

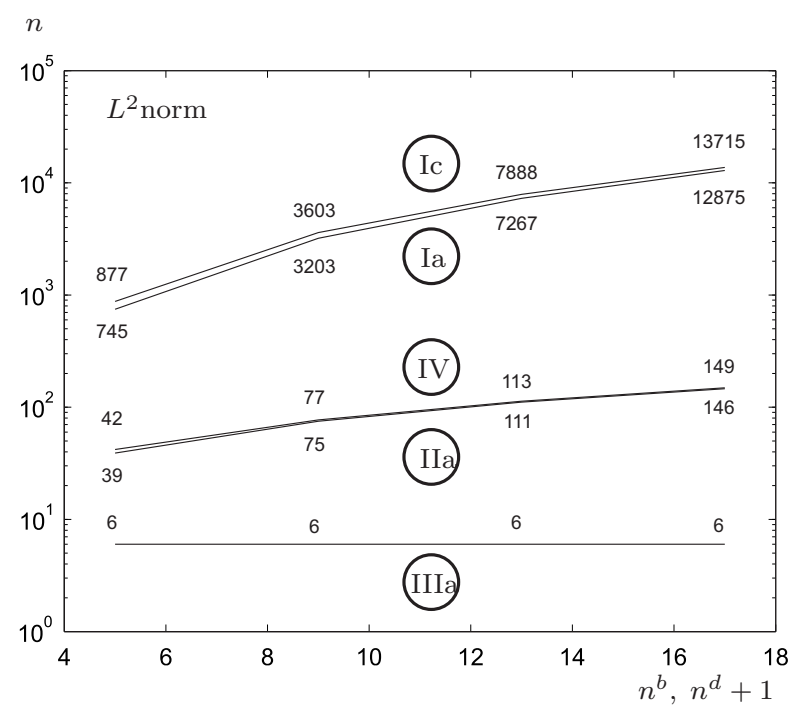

Fig. 16 Number of iterations $n$ vs. number of particles in bridging domain $n^{b}$ and number of particles in pure particle domain $n^{d}=n^{b}-1, L^{2}$ norm in (29)

The influence of the norm used in coupling constraint (29) on the convergence behaviour is obviously insignificant, as a comparison of Figures 16 and 17 is revealing - although the resulting system of equations is known to pose an ill-posed problem in case of the $L^{2}$ norm [2]. Both figures depict the iteration numbers, required by each scheme to fall below a relative error of one percent, versus the particle numbers. The differences are obviously marginal and the additional results for the in between systems having $8 / 9$ and $12 / 13$ particles in the pure particle/bridging domain confirm the trends that have been discussed already. Staggered Scheme IIIa is independent of the particle system size while IV/IIa and Ia/Ic each show a similar behaviour with a slower and faster increase of iteration numbers.

Note that the above comparisons of iteration numbers do not account for the computational costs of each iteration, which are obviously not identical for the different staggering schemes. Nonetheless, since it is the only scheme which seems to be insensitive with respect to the number of particles, we would like to consider Staggering Scheme IIIa as the most interesting option to decouple the computation of this two-scale particlecontinuum simulation method.

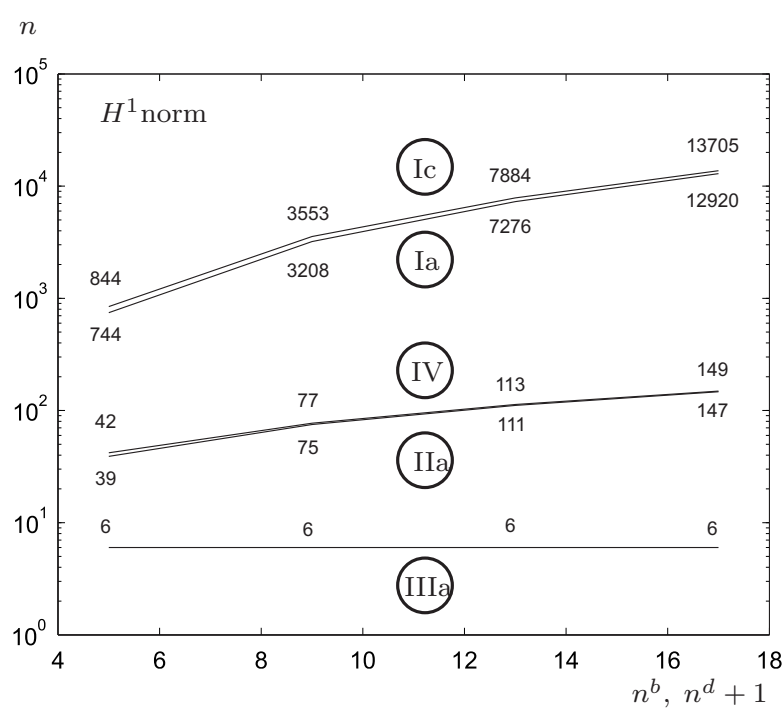

Fig. 17 Number of iterations $n$ vs. number of particles in bridging domain $n^{b}$ and number of particles in pure particle domain $n^{d}=n^{b}-1, H^{1}$ norm in (29)

\section{Conclusions and outlook}

In this contribution, the Arlequin method was used to couple a continuum to a particle domain. Based on the governing equations of this method, the relations for a reduced, one dimensional problem have been derived. First, this problem was solved monolithically using standard solvers for systems of linear equations.

When it comes to larger systems, especially large particle systems, a separated computation of the domains is desirable. However, this requires staggered solution schemes which were systematically derived from the system under consideration and classified according to their characteristics. Numerical tests show that staggered schemes require significant iteration numbers, which strongly depend on the scheme chosen.

Staggered Scheme IIIa seems to be a well suited candidate for an efficient staggered computation of $\mathrm{FE}$ and MD domains. However, it has to be remarked that the investigations done here have to be repeated for $2 \mathrm{~d}$ and $3 \mathrm{~d}$ problems. In particular, it remains to be proved, if the qualitative behavior of staggered schemes also holds for multidimensional approaches. Furthermore, a permutation of boundary conditions, e.g. DiRICHLET instead of Neumann boundary conditions and vice versa, will probably have a significant influence on the iteration numbers required by each scheme. The same will surely hold if the number of FE nodes is modified. However, when it comes to a three-dimensional coupling, the number of particles will clearly dominate in any case. It therefore seems to be reasonable to preferably investigate the influence of the particle domain and to choose 
a staggered scheme that is roughly independent of its size.

It is clear that the adoption of a computational scheme with the minimum number of iteration steps is not decisive in the case of model calculations with a small number of particles and FE nodes. Note however that it is our intention to perform computer simulations on polymer-nanoparticle systems containing $10^{4}$ to $10^{5}$ particles in coarse-grained resolution in the MD domain. Such a system leads to a large computational effort already in standard simulations of the MD type. Even on powerful computers they require at least days of computer time.

In case of coupling to FE system, the required capability of computers becomes even higher. Thus, it is intended to couple not all MD particles, but only 5 to $10 \%$ of the total particle number. More details on that issue are discussed in [15].

In such coupled particle-continuum systems the number of iterative steps between particle and continuum domain determines the feasibility of the suggested hybrid simulations. Test calculations to find the fastest optimization method are an absolute prerequisite in the simulation of real systems.

Acknowledgements We are indebted to Dr. Enrico Riccardi, Azadeh Ghanbari, and Tinashe Ndoro for useful discussion. Financial support by the European Union within the collaborative Nanomodel project (www.nanomodel.eu) is gratefully acknowledged.

\section{References}

1. P. Aubertin, J. Rethore, R. de Borst (2009) Energy conservation of atomistic/continuum coupling. International Journal for Numerical Methods in Engineering 78:1365-1386

2. P.T. Bauman, H. Ben Dhia, N. Elkhodja, J.T. Oden, S. Prudhomme (2008) On the application of the Arlequin method to the coupling of particle and continuum models. Computational Mechanics 42:511-530

3. P.T. Bauman, J.T. Oden, S. Prudhomme (2009) Adaptive multiscale modeling of polymeric materials with Arlequin coupling and Goals algorithms. Computer Methods in Applied Mechanics and Engineering 198:799-818

4. T. Belytschko, S.P. Xiao (2003) Coupling methods for continuum model with molecular model. International Journal for Multiscale Computational Engineering 1:115-126

5. H. Ben Dhia (1998) Problèmes méchaniques multi-échelles: la méthode Arlequin. Comptes Rendus de l'Académie des Science, Series II b 326:899-904

6. H. Ben Dhia, G. Rateau (2001) Analyse mathématique de la méthode Arlequin mixte. Comptes Rendus de l'Académie des Science, Series I 332:649-654

7. H. Ben Dhia, G. Rateau (2005) The Arlequin method as a flexible engineering design tool. International Journal for Numerical Methods in Engineering 62:1442-1462

8. H. Ben Dhia, N. Elkhodja, F.X. Roux (2008) Multimodeling of multi-alterated structures in the Arlequin framework.
Solution with a Domain-Decomposition solver . European Journal of Computational Mechanics 17:969-980

9. L. Chamoin, J.T. Oden, S. Prudhomme (2008) A stochastic coupling method for atomic-to-continuum Monte-Carlo simulations. Computer Methods in Applied Mechanics and Engineering 197:3530-3546

10. R. Delgado-Buscalioni, K. Kremer, M. Praprotnik (2008) Concurrent triple-scale simulation of molecular liquids. The Journal of Chemical Physics 128:114110

11. C. Farhat, F.X. Roux (1991) A method of Finite Element tearing and interconnecting and its parallel solution algorithm. International Journal for Numerical Methods in Engineering 32:1205-1227

12. P.-A. Guidault, T. Belytschko (2007) On the $L^{2}$ and $H^{1}$ couplings for an overlapping domain decomposition method using Lagrange multipliers. International Journal for Numerical Methods in Engineering 70:322-350

13. R. Khare, S.L. Mielke, J.T. Paci, S. Zhang, R. Ballarini, G.C. Schatz, T. Belytschko (2007) Coupled quantum mechanical/molecular mechanical modeling of the fracture of defective carbon nanotubes and graphene sheets. Physical Review B 75:075412

14. R. Khare, S.L. Mielke, G.C. Schatz, T. Belytschko (2008) Multiscale coupling schemes spanning the quantum mechanical, atomistic forcefield, and continuum regimes. Computer Methods in Applied Mechanics and Engineering 197:31903202

15. M. Rahimi, S. Pfaller, G. Possart, P. Steinmann, M.C. Böhm, F. Müller-Plathe (2010) Stochastic boundary conditions to couple particle and continuum domains in the simulation of amorphous materials. Manuscript in preparation

16. M. Schmidt (2006) Minimization toolbox "minFunc", available as Matlab code at http://www.cs.ubc.ca/ schmidtm

17. E.B. Tadmor, M. Ortiz, R. Phillips (1996) Quasicontinuum analysis of defects in solid. Philosophical Magazine A 73(6):1529-1563

18. S.P. Xiao, T. Belytschko (2004) A bridging domain method for coupling continua with molecular dynamics. Computer Methods in Applied Mechanics and Engineering 193:16451669

19. M. Xu, T. Belytschko (2008) Conservation properties of the bridging domain method for coupled molecular/continuum dynamics. International Journal for Numerical Methods in Engineering 76:278-294

20. S. Zhang, R. Khare, Q. Lu, T. Belytschko (2007) A bridging domain and strain computation method for coupled atomistic-continuum modelling of solids. International Journal for Numerical Methods in Engineering 70:913-933 